\title{
Lernergebnisse und individuelle Prozesse des Physik-Lernens mit auditiven und visuellen Hinweisen
}

\author{
Maleen Hurzlmeier ${ }^{1} \oplus$, Bianca Watzka², Christoph Hoyer², Raimund Girwidz² \\ und Bernhard Ertl ${ }^{1}$
}

\author{
Universität der Bundeswehr München, Deutschland \\ ${ }^{2}$ Ludwig-Maximilians-Universität München, Deutschland
}

\begin{abstract}
Zusammenfassung: Die vorliegende Studie untersuchte im Kontext einer videobasierten Lernumgebung, inwieweit Personen durch die Darbietung von auditiven und visuellen Hinweisen beim Lernen komplexer Inhalte unterstützt werden können. Vor dem Hintergrund von Theorien des multimedialen Lernens wurden die Wirkungen der Hinweise auf kognitive Belastung, Lernerfolg sowie Blickbewegungen der Lernenden analysiert. In einem experimentellen Zwei-Gruppen-Design mit Vor- und Nachtest lernten 46 Teilnehmerinnen und Teilnehmer mithilfe visueller $(n=$ 23) oder auditiver $(n=23)$ Unterstützung. Den Lerngegenstand bildeten Skizzen elektrischer Schaltungen, die als kognitives Werkzeug zur Lösung physikalischer Aufgaben eingesetzt werden. Es ergaben sich zwischen den Versuchsbedingungen keine signifikanten Unterschiede in Belastungserleben und Lernerfolg $\left(.00 \leq \eta_{p}{ }^{2} \leq .08\right)$. Allerdings waren Maße der kognitiven Belastung signifikante Prädiktoren des Lernerfolgs. Es zeigten sich auch bedeutsame Einflüsse des Vorwissens auf Wissensabruf $\left(R^{2}=.213\right)$ und Wissenstransfer $\left(R^{2}=.257\right)$. Im Rahmen qualitativer Auswertungen wurden zudem die Blickpfade von Personen mit niedrigem und hohem Vorwissen verglichen. Hier zeigte sich in beiden Modalitäten bei Personen mit niedrigem Vorwissen eher ein detailorientiertes Blickmuster auf Einzelelemente der Schaltungen und bei Personen mit hohem Vorwissen eher ein ganzheitlich orientiertes Blickmuster über das System von Schaltungen. In Bezug auf die vorwissensabhängigen Unterschiede in den Blickmustern und die Komplexität des Lerngegenstandes erscheinen sowohl eine zeitliche als auch eine instruktionale Adaptierbarkeit der Lernumgebung für Lernende mit wenig Vorwissen in beiden Hinweismodalitäten sinnvoll. Für die zukünftige Forschung bietet es sich im Sinne eines Mixed-Method-Ansatzes an, Prozesse und Ergebnisse des Lernens stärker aufeinander zu beziehen, um zu tieferen Erkenntnissen über die Nutzung und Wirkung instruktionaler Hinweise zu gelangen.
\end{abstract}

Schlüsselwörter: Auditive und visuelle Hinweise, Multimedia, Blickbewegungen, Vorwissen, Lernerfolg

Learning outcomes and individual processes of physics learning with auditory and visual cues

\begin{abstract}
In the context of a video-based learning environment, the present study examined how to support learners by auditory and visual instructional cues in learning complex materials. Against the background of theories of multimedia learning, we analyzed the effects of cues on cognitive load, learning outcomes, and eye movements. In an experimental between-subjects design with pre- and post-test, 46 participants learned by the support of instructional visual $(n=23)$ or auditory $(n=23)$ cues. The learning environment consisted of diagrams of electrical circuits, which were used as cognitive tools for solving physical tasks. There were no significant differences in cognitive load and learning outcomes $\left(.00 \leq \eta_{p}{ }^{2} \leq .08\right)$ between the experimental conditions. However, measures of cognitive load were significant predictors of the learning outcomes. In addition, there were significant influences of prior knowledge on retention $\left(R^{2}=.213\right)$ and transfer of knowledge $\left(R^{2}=.257\right)$. A qualitative study delved deeper into gaze pattern of persons with low and high prior knowledge. Here, we found similar effects for both modalities: learners with low prior knowledge tended to show a more detail-oriented gaze pattern towards single elements of the circuits and learners with high prior knowledge tended to show a gaze pattern towards the circuits as a whole. With regard to the differences in the gaze pattern depending on prior knowledge and the complexity of the learning object, both a temporal and an instructional adaptability of the learning environment for learners with low prior knowledge in both modalities appear meaningful. For future research, it seems to be useful to relate the processes and results of learning in terms of a mixed-method approach to gain deeper insight into the use and effects of instructional cueing.
\end{abstract}

Keywords: Auditive and visual cueing, multimedia, eye-tracking, prior knowledge, learning outcomes

\section{Einleitung}

Im Kontext des multimedialen Lehrens und Lernens sind visuelle Repräsentationen grundsätzlich von Bedeutung. Sie dienen neben der inhaltlichen Veranschaulichung als kognitive Werkzeuge der Lösung von domänenspezifischen Aufgabenstellungen. In naturwissenschaftlichen Domänen werden beispielsweise häufig schematische Diagramme eingesetzt (Hegarty, Carpenter \& Just, 1991), um komplexe Informationen zu organisieren sowie abs- 
trakte Phänomene und Konzepte besser darzustellen (Cook, 2006).

Lernende haben jedoch allgemein Schwierigkeiten mit der Interpretation von schematischen Darstellungen (Hegarty et al., 1991). Um den Inhalt einer Darstellung adäquat erfassen und anwenden zu können, müssen Lernende die entsprechenden Konventionen sowie die interagierenden Komponenten des dargestellten Sachverhalts kennen (Butcher, 2006). Hierbei benötigen vor allem Lernende mit geringem Vorwissen instruktionale Unterstützung.

Instruktionale Unterstützung in multimedialen Lernumgebungen kann unter anderem durch die Darbietung von Hinweisen erfolgen. Dabei können Hinweise in unterschiedlichen Modalitäten in das Lernmaterial implementiert werden (z. B. Glaser \& Schwan, 2015; Klein, Viiri \& Kuhn, 2019; Saß, Schütte \& Lindner, 2017; Wang, Lin, Han \& Spector, 2020). Speziell computerbasierte Lernumgebungen ermöglichen neben der Integration von visuellen Hinweisen auch die Darbietung von auditiven Hinweisen (z. B. Glaser \& Schwan, 2015, 2020; Watzka, Hoyer, Ertl \& Girwidz, 2021; Xie, Mayer, Wang \& Zhou, 2019).

Die vorliegende Studie untersucht im Kontext einer videobasierten Lernumgebung, inwieweit Personen durch die Darbietung von auditiven und visuellen Hinweisen beim Lernen komplexer Inhalte unterstützt werden können. Im Rahmen qualitativer Auswertungen vergleicht sie darüber hinaus das Blickverhalten von Lernenden mit hohem und niedrigem Vorwissen. Den Lerngegenstand bilden elektrische Schaltungen, die in der Physik als Schaltskizzen dargestellt werden. Diese gelten als repräsentativ für schematische Darstellungen komplexer Informationen.

\section{Theoretischer Hintergrund}

\section{Theorien des multimedialen Lernens}

Für die Untersuchung der Effektivität von auditiven und visuellen Hinweisen zu visuellen Darstellungen in videobasierten Lernumgebungen sind die Theorie der kognitiven Belastung (Cognitive Load Theory, CLT; Sweller, van Merriënboer \& Paas, 1998) und die kognitive Theorie des multimedialen Lernens (Cognitive Theory of Multimedia Learning, CTML; Mayer, 2014) von Bedeutung (z. B. Glaser \& Schwan, 2020).

Die Theorie der kognitiven Belastung geht von der Annahme einer begrenzten Kapazität des Arbeitsgedächtnisses während des Informationsverarbeitungsprozesses aus (Sweller et al., 1998). Wird während des Lernens oder der Aufgabenbearbeitung die angenommene Verarbeitungskapazität überschritten, so kann die Informationsverar- beitung aufgrund einer kognitiven Überlastung (cognitive overload) beeinträchtigt werden und der Lernerfolg gefährdet sein (Leppink, van Gog, Paas \& Sweller, 2015). Gemäß der Theorie werden drei Arten kognitiver Belastung (cognitive load) unterschieden, die zusammengenommen zur Überlastung des Arbeitsgedächtnisses führen können. Dabei handelt es sich um (1) die inhaltsbedingte kognitive Belastung (intrinsic cognitive load), die sich durch die Komplexität, d.h. die Anzahl interagierender Elemente und der wahrgenommenen Schwierigkeit des Lerninhaltes aufgrund von Vorwissen definiert, (2) die sachfremde kognitive Belastung (extraneous cognitive load), die sich durch die Verarbeitung von zusätzlichen Elementen des Lernmaterials ergibt und (3) die lernrelevante kognitive Belastung (germane cognitive load), die aus dem Interpretieren und Verstehen des Lerninhaltes resultiert (Leppink et al., 2015; deutsche Terminologie der Belastungsarten nach Leutner, Opfermann \& Schmeck, 2014).

Aus dem Forschungsstand zur kognitiven Belastung kann ein positiver Einfluss der lernrelevanten kognitiven Belastung (H1a) sowie ein negativer Einfluss der sachfremden kognitiven Belastung auf den Lernerfolg erwartet werden (H1b).

Im Zusammenhang mit multimedialen Darbietungen beschreibt die kognitive Theorie des multimedialen Lernens drei zentralen Annahmen: (1) die kognitive Verarbeitung von visuellen und auditiven Informationen erfolgt anhand von zwei weitgehend getrennten Verarbeitungskanälen, (2) jeder dieser Kanäle verfügt über eine begrenzte Verarbeitungskapazität und (3) die Informationsverarbeitung verläuft als aktiver Prozess, bei dem multimedial präsentierte Informationen ausgewählt, organisiert und unter Einbezug des Vorwissens in Beziehung zueinander gesetzt werden (Mayer, 2014).

\section{Hinweise in multimedialen Lernumgebungen}

Die meisten Lernenden profitieren von expliziten Anleitungen während des Lernens von Naturwissenschaften (Kirschner, Sweller \& Clark, 2006). Der Vorteil der Anleitung beginnt erst dann zu schwinden, wenn die Lernenden über ausreichend hohes Vorwissen verfügen, um sich selbst eine ,interne“ Anleitung zu geben. Eine Möglichkeit der expliziten Anleitung ist das Geben instruktionaler Hinweise.

Allgemein gesprochen erweisen sich Hinweise insbesondere beim Lernen komplexer Inhalte aus visuellen Darstellungen als hilfreich. Sie können die Organisation des Lerninhaltes betonen oder die Beziehungen relevanter Aspekte im Lernmaterial hervorheben (de Koning, Tabbers, Rikers \& Paas, 2009). Darüber hinaus ermöglichen 
Hinweise die Aufmerksamkeit der Lernenden auf die relevanten Aspekte im Lernmaterial zu lenken (z.B. Brünken, Seufert \& Zander, 2005; de Koning et al., 2009; Ertl, Fischer \& Mandl, 2006; Signalisierungsprinzip; van Gog, 2014), um das Ausmaß der visuellen Suche zu reduzieren.

Metaanalytische Ergebnisse konnten belegen, dass Hinweise in Lernmaterialien unterschiedlicher Domänen die visuelle Aufmerksamkeit lenken und grundsätzlich den Lernerfolg verbessern (z. B. Richter, Scheiter \& Eitel, 2015; Schneider, Beege, Nebel \& Ray, 2018). Schneider et al. (2018) fanden beim Einsatz von Hinweisen in multimedialen Lernmaterialien positive Effekte mittlerer Stärke auf den Wissensabruf. Richter et al. (2016) konnten - ebenso beim Einsatz von Hinweisen in multimedialen Lernmaterialien - kleine bis mittlere positive Effekte auf Verständnis und Wissenstransfer nachweisen.

Aus Sicht der CLT kann durch die Hinweisgabe zunächst die sachfremde Belastung verringert werden, da diese die Aufmerksamkeit der Lernenden auf die relevanten Aspekte der Lernumgebung lenken. Dadurch bleiben mehr Ressourcen für das Verstehen und Interpretieren des Lerngegenstandes erhalten (Glaser \& Schwan, 2020; de Koning, Tabbers, Rikers \& Paas, 2010). Xie et al. (2017) fanden in ihrer Metaanalyse einen geringen negativen Effekt der Hinweisgabe auf die gesamte kognitive Belastung. Sank die kognitive Belastung durch Hinweise, verbesserten sich die Behaltens- und Transferleistungen der Lernenden signifikant. Zu einem ähnlichen Ergebnis kamen auch Klein et al. (2019), die für das Lernen mit Hinweisen im Kontext der Physik eine höhere Transferleistung sowie eine niedrigere mentale Belastung nachweisen konnten.

Die hilfreichen Wirkungen von Hinweisen konnten jedoch in einzelnen Untersuchungen nicht immer bestätigt werden (z. B. Crooks, Cheon, Inan, Ari \& Flores, 2012; de Koning et al., 2010; Kriz \& Hegarty, 2007). Hinweise sorgen zwar dafür, dass in einer ersten Verarbeitungsstufe die Aufmerksamkeit neu ausgerichtet wird, aber es müssen weitere Verarbeitungsprozesse erfolgen, um einen Sachverhalt vollkommen zu verstehen (Scheiter \& Eitel, 2015). Hinweise verursachen eine zusätzliche lernbezogene Belastung, da sie verarbeitet und in die kognitiven Schemata der Lernenden integriert werden müssen (vgl. Huk, Steinke \& Floto, 2010; Schneider et al., 2018; Seufert \& Brünken, 2006). Sind die Informationen aus den Hinweisen für die Lernenden dann nicht hilfreich, induzieren diese eine sachfremde Belastung, was den Lernerfolg beeinträchtigt (Brünken et al., 2005; vgl. auch Leppink et al., 2015). Auch die intrinsische Belastung steigt, weil insbesondere inhaltsunterstützende Hinweise die Komplexität des Lernmaterials erhöhen (Cook, 2006; vgl. auch Leppink et al., 2015).

Darüber hinaus wird der moderierende Einfluss des Vorwissens im Kontext des Einsatzes von Hinweisen in multimedialen Lernumgebungen diskutiert. Für Personen mit hohem Vorwissen kann sich eine höhere kognitive Belastung ergeben, da sich die Hinweise als überflüssig und störend erweisen (Cook, 2006). Für Personen mit niedrigem Vorwissen dagegen kann sich durch die Hinweisgabe eine Reduzierung der kognitiven Belastung ergeben, da ihre Aufmerksamkeit auf die lernrelevanten Aspekte gelenkt wird (Cook, 2006). Die Befundlage zum moderierenden Effekt des Vorwissens auf den Lernerfolg ist uneinheitlich. Richter et al. (2016) konnten die Wirksamkeit der Hinweisgabe insbesondere für Lernende mit niedrigem Vorwissen feststellen. Jedoch ergab sich bei Schneider et al. (2018) kein moderierender Effekt des Vorwissens auf den Lernerfolg. Aus zahlreichen Studien (z. B. Ertl, Kopp \& Mandl, 2005; Hattie, 2009) ist jedoch bekannt, dass das Vorwissen generell einen starken Prädiktor für den Lernerfolg darstellt.

(H2) Ausgehend davon wird erwartet, dass sich das Vorwissen auch im Rahmen dieser Studie als ein starker Prädiktor für den Lernerfolg erweist.

\section{Auditive und visuelle Hinweise in multimedialen Lernumgebungen und evidenzbasierte Implikationen}

Eine Klassifikationsmöglichkeit der Hinweise ergibt sich auf der theoretischen Grundlage des multimedialen Lernens nach Mayer (2014). Dabei lässt sich modalitätsorientiert zwischen auditiven und visuellen Hinweisen unterscheiden (z.B. Xie et al., 2019). Auditive Hinweise beinhalten klangliche Darbietungen (z.B. Signaltöne oder Intonation) sowie gesprochene Texte oder Sätze zum Lernmaterial, die über den auditiven Kanal wahrgenommen und verarbeitet werden. Visuelle Hinweise schließen alle bild- und textbasierenden Möglichkeiten ein (z.B. Pfeile, geschriebene Sätze oder farbliche Hervorhebungen), die über den visuellen Kanal aufgenommen und verarbeitet werden.

Beruhend auf den Annahmen der CTML konnten zahlreiche Prinzipien und Effekte des multimedialen Lernens abgeleitet und empirisch nachgewiesen werden (für einen umfassenden Überblick siehe z. B. Mayer, 2014). So kann neben dem bereits erwähnten Signalisierungsprinzip auch der Modalitätseffekt zur Erklärung der Wirkungsweise von auditiven und visuellen Hinweisen zu visuellen Repräsentationen herangezogen werden. Dem Modalitätseffekt zufolge kommt es aufgrund der Darbietung von auditiven und visuellen Informationen $\mathrm{zu}$ einem höheren Lernerfolg als bei Darbietungen in ausschließlich einer Modalität. Da die Kapazitäten beider Verarbeitungskanäle genutzt werden, kann eine kognitive Überlastung bei der Nutzung nur eines Kanals vermieden werden (Low \& Sweller, 2014). 
Beide Hinweismodalitäten können in multimedialen Lernumgebungen sowohl lernförderlich als auch lernhinderlich sein. Aus zahlreichen Experimenten zum Modalitätseffekt ist bekannt, dass die Substituierung von geschriebenem durch gesprochenen Text die Lernleistungen verbessert (z. B. Brünken et al., 2005; Kühl, Scheiter, Gerjets \& Edelmann, 2011; Mayer \& Moreno, 1998). Übertragen auf die modalitätsspezifische Hinweisgestaltung würde dies bedeuten, dass auditive Hinweise den Lernerfolg stärker begünstigen können als visuelle Hinweise, da eine Überlastung des visuellen Kanals vermieden werden kann. In Studien zu naturwissenschaftlichen Lerninhalten konnten Modalitätseffekte auch für Lerninhalte mit hoher Elementinteraktivität (Tindall-Ford, Chandler \& Sweller, 1997) und in Abhängigkeit vom Vorwissen (Kalyuga, Chandler \& Sweller, 2000) nachgewiesen werden.

(H3) Ausgehend vom Modalitätseffekt und den beschriebenen Befunden wird im Rahmen der vorliegenden Studie erwartet, dass Lernende durch auditive Hinweise einen höheren Lernerfolg als Lernende mit visuellen Hinweisen erzielen.

Bei der visuellen Hinweisgabe kann sich zudem bei komplexen Lerninhalten und niedrigem Vorwissen eine Überlastung des visuellen Kanals ergeben (vgl. Kalyuga, 2012). Tabbers, Martens und van Merriënboer (2004) fanden jedoch, dass visuelle Hinweise zu Bildmaterialien $\mathrm{zu}$ einer besseren Behaltensleistung führten, während die Substitution von geschriebenem zu gesprochenem Text eine niedrigere Transfer- und Behaltensleistung nach sich zog.

Auditive Repräsentationen sind transient (Mayer, 2014), was bedeutet, dass insbesondere gesprochene Inhalte auditiver Hinweise länger im Arbeitsgedächtnis aufrechterhalten werden müssen, während das Bildmaterial auf die in den Hinweisen genannten Aspekte abgesucht wird. Die dabei entstehende kognitive Belastung ist abhängig von der Komplexität und Länge der auditiven Darbietung (Kalyuga, 2012). Im Gegensatz dazu sind visuelle Repräsentationen persistent (Mayer, 2014), was bedeutet, dass visuelle Hinweise bei Bedarf wiederholt betrachtet werden können. Dadurch kann zwar die visuelle Aufmerksamkeit der Lernenden mehrmals auf die relevanten Aspekte im Lernmaterial gelenkt werden (vgl. Repräsentationale Führung; Suthers \& Hundhausen, 2003), zugleich aber die lernbezogene Belastung erhöht werden (vgl. Leppink, Paas, van der Vleuten, van Gog \& van Merriënboer, 2013).

(H4) Die Persistenz der visuellen Hinweise und die daraus resultierende Möglichkeit einer mehrfachen Verarbeitung lässt im Rahmen der vorliegenden Untersuchung annehmen, dass die lernrelevante Belastung durch visuelle Hinweise höher ausfällt als durch auditive Hinweise.
Wenn auditive oder visuelle Hinweise zur Unterstützung des Lernens von Bildmaterialien gegeben werden, müssen Informationen aus verschiedenen Quellen miteinander integriert werden. Hier erfahren auditive Hinweise einen Vorteil, da gesprochener Text simultan mit der piktoralen Information verarbeitet werden kann (z.B. Rummer, Fürstenberg \& Schweppe, 2008; Seufert, Schütze \& Brünken, 2009; Tabbers et al., 2004). Jedoch erfordern verbale Informationen einen Transformationsprozess, da das Gehörte (z.B. auditive Referenzen oder Beschreibungen) in ein mentales Modell überführt werden muss, wodurch sich die sachfremde Belastung erhöht (Leppink et al., 2015). Bei visuellen Hinweisen ist der Lernende dagegen im Sinne eines Split-AttentionEffekts (siehe Ayres \& Sweller, 2014) gezwungen, die Aufmerksamkeit abwechselnd auf Bildinformation und visuellen Hinweis zu richten, was ebenso die sachfremde Belastung erhöht (vgl. Seufert et al., 2009).

In Abwägung der Effekte beider Hinweismodalitäten auf die sachfremde Belastung, sollte die visuelle Hinweisgabe aufgrund einer repräsentationalen Führung (Suthers \& Hundhausen, 2003) disambiguierend wirken und dadurch die Aufmerksamkeit eindeutiger auf die relevanten Elemente lenken als die auditive Hinweisgabe.

(H5) Daher sollte durch die visuelle Hinweisgabe eine geringere sachfremde Belastung entstehen als durch die auditive.

\section{Eye-Tracking zur Untersuchung multimedialer Lernumgebungen}

Über das Eyetracking-Verfahren lässt sich zeigen, wie sich der Wechsel der Aufmerksamkeit zwischen verschiedenen visuell wahrnehmbaren Informationsquellen vollzieht (Hyönä, 2010). Im Allgemeinen werden zwei Arten von Blickbewegungsmaßen erhoben: Fixationen, welche die Ruhepunkte des Auges auf dem Lernmaterial bezeichnen und Sakkaden, welche die Bewegungen des Auges zwischen den Ruhepunkten kennzeichnen (van Gog \& Jarodzka, 2013). Weitergehend untersucht werden dabei zum Beispiel deren Anzahl, Dauer oder Länge. Zahlreiche Studien analysieren den Zusammenhang der Informationsverarbeitungsprozesse gemäß der CTML (Mayer, 2014) mit Blickbewegungen in multimedialen Lernumgebungen. Vermehrte Sakkaden zwischen visuellen Elementen deuten auf die Zusammenführung von Informationen hin (Schüler, 2017; integration process; Mayer, 2014). Längere Fixationsdauern auf visuelle Elemente sprechen für eine stärkere Organisation und Verarbeitung von Informationen (Schüler, 2017; organization process; Mayer, 2014). Diese Parameter werden jedoch in einigen Studien in Abhängigkeit vom Lernsetting abweichend interpretiert (für einen umfassenden Überblick siehe Alemdag \& Cagiltay, 2018). 
Die Blickbewegungsparameter können sowohl auf globaler als auch auf lokaler Ebene ermittelt werden (vgl. Holmqvist, Nyström, Andersson, Dewhurst, Jarodzka \& van der Weijer, 2011; Raney, Campbell \& Bovee, 2014). Globale Maße spiegeln Blickbewegungen über den gesamten Bildschirm wider. Lokale Maße ermöglichen dagegen eine differenzierte Analyse von Blickbewegungen in einzelnen Bereichen (areas of interest) der Lernumgebung.

Die räumliche, zeitliche sowie mengenmäßige Verteilung des Blickverhaltens kann zudem anhand von Blickpfadmustern dargestellt werden. Sie ergeben sich aus einer Folge von Sakkaden innerhalb einer bestimmten Zeitspanne (Holmqvist et al., 2011).

Verschiedene Studien haben bereits den Einsatz von Hinweisen in multimedialen Lernumgebungen anhand von Blickbewegungsmessungen untersucht (z. B. de Koning et al., 2010; Glaser \& Schwan, 2015; Klein et al., 2019; Saß et al., 2017; Scheiter \& Eitel, 2015; Wang et al., 2020; Watzka et al., 2021; Xie et al., 2019), um Erkenntnisse über die Wirkung der Hinweise zu erhalten. So konnten beispielsweise Saß et al. (2017) anhand von Blickbewegungsanalysen zeigen, dass Hinweise die Aufmerksamkeit generell auf die referenzierten Darstellungen lenken. Klein et al. (2019) konnten belegen, dass Lernende durch Hinweise längere Fixationsdauern und häufigere Fixationen auf den lernrelevanten Bereichen aufweisen.

Darüber hinaus existiert eine Reihe von quantitativen Untersuchungen zum Zusammenhang von Blickbewegungen und Vorwissensunterschieden, die Gegenfurtner, Lethinen und Säljö (2011) metaanalytisch zusammengefasst haben. Die Autoren betrachteten Vorwissensunterschiede im Verständnis von visuellen Darstellungen anhand von Blickbewegungsmessungen. Sie konnten zeigen, dass Personen mit höherem Vorwissen mehr Fixationen auf relevante Informationen, weniger Fixationen auf irrelevante Informationen und längere Sakkaden als Personen mit niedrigerem Vorwissen aufweisen. Yang, Chang, Chien, Chien und Tseng (2013) fanden, dass mit steigendem Vorwissen Personen eine größere Anzahl von Vergleichen zwischen Elementen einer Lernumgebung anstellen. Dabei muss jedoch einerseits der Inhalt und andererseits der Aufbau der zu untersuchenden Lernumgebung berücksichtigt werden.

Eng im Zusammenhang mit den bisherigen metaanalytischen Ergebnissen aus der Expertiseforschung von Gegenfurtner et al. (2011) stehen auch die Erkenntnisse neuerer Studien (z. B. Kekule, 2014; Klein, Becker, Küchemann \& Kuhn, 2021; Klein et al., 2020; Lindner, Eitel, Thoma, Dalehefte, Ihme \& Köller, 2014; Mozaffari Chanijani, Klein, Bukhari, Kuhn \& Dengel, 2016) aus dem naturwissenschaftlichen Bereich, welche insbesondere das Blickverhalten beim Bearbeiten von Aufgaben mit visuellen Darstellungen untersuchten. Beim Vergleich von Experten und Novizen bzw. starken und weniger starken Lernenden ergaben sich deutliche Unterschiede im Blickverhalten. So richteten Personen mit höherer Expertise weniger Aufmerksamkeit auf irrelevante Antwortoptionen (Klein et al., 2020; Lindner et al., 2014), betrachteten grafische Darstellungen ganzheitlicher (Kekule, 2014) und zeigten ein systematischeres Vorgehen bei der Aufgabenbearbeitung (Klein, Viiri, Mozaffari Chanijani, Dengel \& Kuhn, 2018; Mozaffari Chanijani et al., 2016).

\section{Die aktuelle Studie}

Im Gegensatz zur Forschung der visuellen Hinweisgebung gibt es bislang nur vereinzelt Studien (z.B. Glaser \& Schwan, 2015, 2020; Watzka et al., 2021; Xie et al., 2019), die auch den Einsatz von auditiver Hinweisgebung in den Blick nehmen. Zum Einsatz von inhaltsunterstützenden, textbasierten Hinweisen in multimedialen Lernumgebungen existieren zwar bereits einige Studien (z. B. Ertl, Kopp \& Mandl, 2008; Seufert \& Brünken, 2006; Song \& Bruning, 2016; Weinberger, Ertl, Fischer \& Mandl, 2005), für vertiefte Erkenntnisse sind jedoch weitere Studien unter Einbezug des Blickverhaltens der Lernenden erforderlich.

Wang et al. (2020) fordern zudem gerade in videobasierten Lernumgebungen weitere Forschung zum Einfluss von Hinweisgebungen, insbesondere auf kognitive Prozesse und den Lernerfolg. Glaser und Schwan (2020) konstatieren mit Blick auf die in der Forschung verwendeten Lernmaterialien, dass empirische Befunde zu Lernsituationen, in denen ausschließlich visuelle Repräsentationen, die als kognitives Werkzeug dienen, rar sind.

Blickbewegungsmessungen in Verbindung mit räumlichen Analysen sind ein weiteres Feld defizitärer Forschung (Alemdag \& Cagiltay, 2018). Insbesondere fehlen Untersuchungen zu Blickpfadmustern, welche Hinweise auf die Verarbeitung multimedialer Informationen geben (Krejtz, Duchowski, Krejtz, Kopacz \& ChrząstowskiWachtel, 2016). Alemdag und Cagiltay (2018) fordern speziell qualitative Analysen in die Blickbewegungsforschung einzubinden, um quantitative Ergebnisse zu ergänzen. Da quantitative Analysen häufig eine Fokussierung auf den Mittelwert aufweisen, viele Probanden Bereiche aber sehr unterschiedlich betrachten, kann durch qualitative Analysen eine bessere Darstellung des individuellen Lernverhaltens erfolgen.

Zudem werden in der vorliegenden Studie, im Vergleich zu den visuellen Darstellungen der bisher beschriebenen Studien, die Darstellungen verschiedener elektrischer Schaltkreise untersucht. Solche visuellen Repräsentationen beinhalten deutlich mehr interagierende Elemente als beispielsweise die Interpretation von Graphen in einem Koordinatensystem (z.B. Kekule, 2014; Klein et al., 2020) 
oder Repräsentations- sowie Organisationsbilder (z. B. Saß et al., 2017).

Die aktuelle Studie umfasst quantitative und qualitative Fragestellungen. Die quantitativen untersuchen Unterschiede zwischen auditiven und visuellen Hinweisen auf den Lernerfolg und Einflussfaktoren auf den Lernerfolg. Die qualitative Fragestellung fokussiert Prozesse, die sich während der Auseinandersetzung mit den domänenspezifischen Elementen bei Personen mit niedrigem und hohem Vorwissen vollziehen. Dadurch können in Abhängigkeit vom Vorwissen auch Erkenntnisse gewonnen werden, welche Informationen den Lernenden in den Hinweisen zukünftig dargeboten werden müssen.

\section{Fragestellungen}

Konkret lassen sich für die Studie folgende Forschungsfragen ableiten:

1. Inwieweit unterscheiden sich die modalitätsspezifischen Bedingungen in (a) der kognitiven Belastung und (b) in den Lernergebnissen?

Im Rahmen der Forschungsfrage werden die im Theorieteil aufgestellten Hypothesen drei, vier und fünf überprüft.

2. Inwieweit werden die Unterschiede in den Lernergebnissen durch (a) Hinweismodalität, (b) Vorwissen und (c) kognitive Belastung erklärt?

Die Beantwortung dieser Frage erfolgt anhand der Überprüfung der Hypothesen eins und zwei.

3. Inwieweit unterscheiden sich die Blickpfadmuster zwischen Lernenden mit hohem Vorwissen von Personen mit niedrigem Vorwissen innerhalb der beiden Bedingungen?

Diese Frage legt eine qualitative, explorative Analyse der Blickbewegungen nahe, die zudem die Interpretation der quantitativen Ergebnisse zur Hinweisgabe stützen kann.

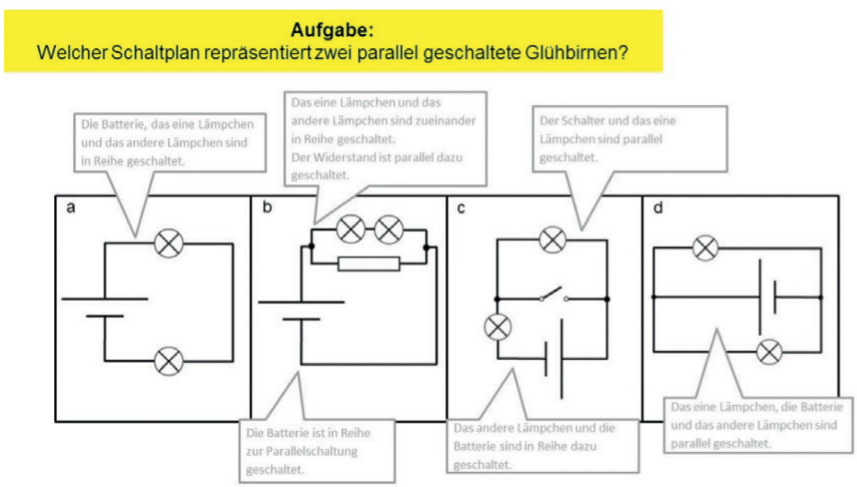

\section{Methode}

\section{Rahmen der Studie}

Die Studie wurde auf Basis eines positiven Votums der lokalen Ethikkommission (datiert vom 29.6.2017) gemäß den Richtlinien der Deutschen Gesellschaft für Psychologie (2018) durchgeführt. Die Teilnahme erfolgte freiwillig und wurde mit einer Aufwandsentschädigung vergütet. Die Versuchsdaten wurden ausschließlich pseudonymisiert auf einem Notebook im Labor erhoben; für die Fragebogendaten wurde zur Erhebung auf das Umfragewerkzeug Unipark/Questback zurückgegriffen, für das eine Datenschutzerklärung vorliegt.

\section{Experimentelles Design}

Der computergestützten Untersuchung lag ein BetweenSubjects-Design mit einem Vor- und Nachtest zugrunde. Der Prätest bestand aus einem mehrteiligen Fragebogen und einem Vorwissenstest, an den sich eine zweigeteilte Lernphase anschloss. Im ersten Abschnitt der Lernphase bearbeiteten die Versuchsteilnehmerinnen und -teilnehmer eine Lehreinheit mit Fotografien von Bauteilen und Symbolen elektrischer Schaltungen mit den jeweiligen Fachtermini (z. B. Messgeräte für Stromstärke oder Lichtquelle), im zweiten Abschnitt lösten sie acht videobasierte Aufgaben mit jeweils vier Antwortalternativen. Diese hatten jeweils eine begrenzte Dauer zwischen eineinhalb und drei Minuten. In diesen acht Aufgaben wendeten die Lernenden entweder Konzepte elektrischer Schaltungen an oder übertrugen elektrische Schaltungen auf physikalisch äquivalente Darstellungen. Fragen zur kognitiven Belastung sowie ein Wissenstest bildeten den Abschluss der experimentellen Studie.

Die experimentelle Intervention erfolgte in der zweiten Lernphase, indem entweder ausschließlich visuelle oder
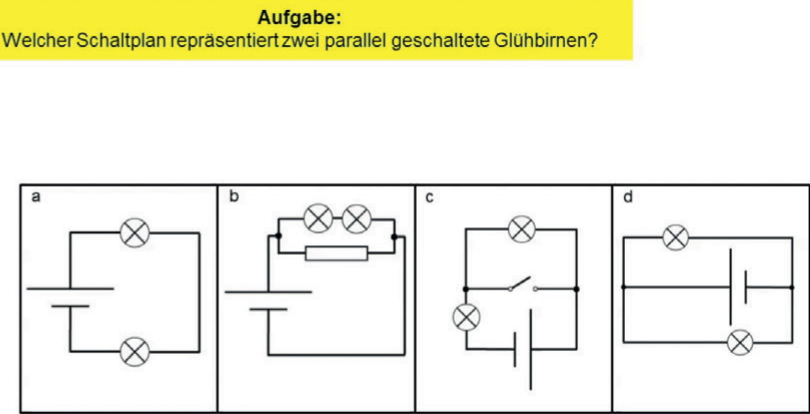

Abbildung 1. Darstellung einer Aufgabe aus dem Lernmaterial. Die linke Abbildung zeigt den Bildschirm bei visueller Hinweisgebung, die rechte Seite präsentiert den Bildschirm bei auditiver Hinweisgebung. 
auditive Hinweise (instructional cues) zum physikalischen Lernmaterial eingesetzt wurden (siehe Abb. 1). In der visuellen Versuchsbedingung waren die Hinweise textuell anhand von rechteckigen Sprechblasen mit Leitpfeilen in das Lernmaterial eingebettet. Im Gegensatz dazu wurden in der auditiven Versuchsbedingung die Hinweise mündlich über Lautsprecher dargeboten und zusätzlich präpositionale Ausdrücke, wie „oben“, „unten“, „links“ oder "rechts“ verwendet, um die Aufmerksamkeit der Probandinnen und Probanden auf den Inhalt der einzelnen Schaltungen zu lenken. In diesem circa 20-minütigen Lernabschnitt wurden in beiden Hinweismodalitäten automatisch ablaufend und in zeitlich äquivalenter Länge exakt dieselben Informationen zu den einzelnen elektrischen Schaltungen vermittelt.

\section{Untersuchungsmaterial und Instrumente}

Im Folgenden werden die für diese Studie relevanten Materialien vorgestellt.

Vorwissen. Die Erhebung des Vorwissens erfolgte durch die Abfrage von grundlegenden Symbolen elektrischer Schaltungen (6 Items; Cronbachs $\alpha=.87$; z. B. „Was stellt dieses Schaltsymbol dar?"). Es wurde sich an in Deutschland üblichen Schaltsymbolen und Bauteilen orientiert, die in schulischen und universitären Physiksammlungen vorkommen. Die Versuchsteilnehmerinnen und -teilnehmer ordneten entweder einem Schaltsymbol oder einer Schaltskizze eine von vier Antwortmöglichkeiten zu. Dieses Faktenwissen zur Symbolik stellt eine wichtige Voraussetzung in der Arbeit mit elektrischen Schaltungen dar.

Kognitive Belastung. Zur Erfassung der mentalen Belastung wurde ein bereits validierter 13-Item-Fragebogen von Leppink, Paas, van Gog, van der Vleuten und van Merriënboer (2014) verwendet. Das Instrument ermöglicht nicht nur die Messung der gesamten mentalen Belastung, sondern auch eine differenzierte Messung der einzelnen mentalen Belastungsarten. Die Items der inhaltsbedingten Belastung (4 Items; Cronbachs $\alpha=.92$; z. B. "Ich investiere eine sehr hohe mentale Anstrengung beim Betrachten der komplizierten Videos."), der sachfremden Belastung (4 Items; Cronbachs $\alpha=.79$; z. B. „Die Erklärungen und Anweisungen in den Videos waren sehr unklar.") und der lernrelevanten Belastung (5 Items; Cronbachs $\alpha=$ .92; z.B. „Das Betrachten der Videos verbesserte wirklich mein Verständnis vom gezeigten Inhalt.") wurden auf einer zehnstufigen Likertskala gemessen $(1=$ stimmt gar nicht bis 10 = stimmt genau).

Lernerfolg. Der Lernerfolg der Studienteilnehmerinnen und -teilnehmer wurde anhand von 21 MultipleChoice-Items mit jeweils vier Schaltskizzen als Antwortmöglichkeiten gemessen (z.B. „In welcher Schaltung brennt die Lampe gleich hell, wie in der oben gezeigten?“). Der standardisierte Test „Determining and Interpreting Resistive Electric Circuit Concept Test" (DIRECT) von Engelhart und Beichner (2004) bildete die Grundlage des Lernerfolgstests. Allerdings waren Adaptionen zur Gewährleistung einer optimalen Passung zur Lernumgebung nötig, da im Originaltest unter anderem amerikanische Symbolik und Teils andere Fachinhalte erfasst werden. Sieben der eingesetzten Items erfassten den Wissensabruf (Cronbachs $\alpha=.58$ ) und beinhalteten Schaltungen, die bereits in der Lernphase zuvor behandelt worden waren. Die anderen 14 Items erforderten einen Wissenstransfer auf neue Schaltungen (Cronbachs $\alpha=.71$ ). Durch die Ähnlichkeit dieser Items mit den Lernaufgaben ist davon auszugehen, dass diese Skala primär den nahen Transfer erfasste.

Um die Validität der Items zum Vorwissen und Lernerfolg sicherzustellen, wurden sie von Physikdidaktikern und Lehr-Lernforschern gemeinsam entwickelt und überprüft. Beide Tests erfassten die in Bildungsplänen und Schulbüchern klassisch vertretenen Lerninhalte der Elektrizitätslehre - maßgeblich die Lerninhalte der Mittelstufe. Die im Lernerfolgstest gezeigten Schaltungen decken einfache Reihen-, Parallel- und gemischte Schaltungen bestehend aus mindestens 2 und maximal 5 Bauteilen ab.

\section{Stichprobe}

Die Datengrundlage der Studie bildete eine Convenience-Stichprobe von $N=46$ deutschen Universitätsangehörigen ( $n=12$ weiblich, $n=33$ männlich, $n=1$ divers) im Alter zwischen 20 und 35 Jahren, die im Kontext von Seminaren gewonnen werden konnte. Die Repräsentativität der Stichprobe ist damit vergleichbar mit einer Vielzahl pädagogisch-psychologischer Studien. Das durchschnittliche Alter der Stichprobe lag bei $M=24.11$ Jahren $(S D=3.80)$. Der überwiegende Teil der Probandinnen und Probanden (41) stammten aus dem bildungswissenschaftlichen Bereich und 5 aus technischen Bereichen. Diese wurden durch einen Versuchsleiter zufällig auf die beiden Bedingungen verteilt $(n=23$ visuell und $n=23$ auditiv). Aus den technischen Bereichen befanden sich 4 Personen in der auditiven und 1 Person in der visuellen Versuchsbedingung. Obwohl die Personen aus den technischen Bereichen signifikant bessere Leistungen im Vorwissenstest erzielten $(t(44)=2.09, p=.042)$, konnten im Vergleich der beiden Versuchsbedingungen keine signifikanten Unterschiede im Vorwissen $(t(44)=$ $0.96, p=.341)$ oder im Interesse am Fach Physik $(t(44)=$ $1.11, p=.272)$ festgestellt werden. Einige Personen aus beiden Bereichen konnten die maximale Punktzahl im Vorwissenstest erreichen. 


\section{Durchführung}

Das Experiment wurde innerhalb eines Zeitraums von mehreren Wochen als Einzeluntersuchung in Anwesenheit eines geschulten Versuchsleiters durchgeführt und fand vollständig am Computer eines Medienlabors statt. $\mathrm{Zu}$ Beginn wurden die Teilnehmerinnen und Teilnehmer über den Versuch und den Versuchsablauf aufgeklärt, sodass sie informiert einer Teilnahme zustimmen konnten.

Nach der Generierung eines persönlichen Pseudonyms (nach DGPS, 2018, S.126) wurde zur Beantwortung eines Fragebogens mit verschiedenen Fragen über den persönlichen Hintergrund der Lernenden und des Vorwissenstests aufgefordert. Im Anschluss daran erfolgte die zweiteilige Lernphase über elektrische Schaltungen. Die erste Lernphase war für alle Lernenden gleich und frischte das für den Umgang mit den Schaltungen erforderliche Symbolwissen auf. Im Anschluss wurde die Eye-Tracking-Software durch den Versuchsleiter gestartet sowie die Einstellung und Kalibrierung des Eye-Trackers vorgenommen, um die Blickbewegungen auf dem Monitor kontaktlos aufzuzeichnen. In der zweiten Lernphase erfolgte das Lernen in einer der beiden Versuchsbedingungen. Die Messung der kognitiven Belastung fand unmittelbar nach der zweiten Lerneinheit statt. Abschließend wurde der Lernerfolg der Versuchsteilnehmerinnen und -teilnehmer anhand von Wissensabruf- und Wissenstransferaufgaben erhoben. Insgesamt dauerte das gesamte Experiment etwa eine Zeitstunde.

\section{Analyse des Blickverhaltens}

Das Blickverhalten der Probandinnen und Probanden wurde mit einer Distanz von ungefähr 60 Zentimeter Abstand zu einem 15-Zoll-Bildschirm vom Eye-Tracker SMI-RED aufgezeichnet und mittels der Software BeGaze Version 3.7 ausgewertet. Die Messgrundlage bildeten acht gruppenspezifische Videos in der zweiten Lernphase. Für die qualitative Analyse der visuellen Aufmerksamkeit wurden Blickpfade generiert.

\section{Ergebnisse}

Nachfolgende Analysen wurden anhand von SPSS mit der Version 26 durchgeführt. Für die verwendeten Rechenverfahren waren alle erforderlichen Bedingungen erfüllt.

\section{Deskriptive Statistik}

Tabelle 1 zeigt modalitätsunspezifisch die Mittelwerte sowie die ermittelten Extremwerte der untersuchten Variablen. Bei den Wissenstests zeigt sich mit Werten um die .70, dass die Lernenden etwa 70 Prozent der Aufgaben richtig lösen konnten. Von den drei Arten kognitiver Belastung dominiert die lernbezogene Belastung, während intrinsische und extrinsische Belastung vergleichsweise geringer ausfallen. Das theoretische Maximum zur Ermittlung der kognitiven Belastung wurde in keiner der drei Belastungsarten erreicht.

\section{Überprüfung der Versuchsbedingungen}

Um die Wirkung der auditiven sowie visuellen Bedingung zu überprüfen, werden zuerst Unterschiede in den Blickparametern zwischen den beiden Bedingungen betrachtet. Die Ergebnisse zeigen auf globaler Ebene (Gesamtbildschirm), dass es signifikante Unterschiede in der Anzahl der Sakkaden und Fixationen sowie in der durchschnittlichen Fixationsdauer (siehe Tabelle E5 im Elektronischen Supplement 1) zwischen den Bedingungen gab. Auch auf lokaler Ebene, wenn man nur den Bereich der Schaltskizzen betrachtet (siehe Tabelle E6 sowie Abbildungen E4 und E5 im ESM 1), traten diese

Tabelle 1. Deskriptive Statistik zu Vorwissen, kognitiver Belastung und Lernerfolg

\begin{tabular}{|c|c|c|c|c|c|}
\hline Variable & Itemanzahl & M & $S D$ & Spannweite & Mögliche Spannweite \\
\hline Vorwissen & 6 & 0.70 & 0.36 & $0.00-1.00$ & $0.00-1.00$ \\
\hline \multicolumn{6}{|l|}{ Kognitive Belastung } \\
\hline inhaltsbedingte Belastung & 4 & 2.80 & 1.84 & $1.00-7.50$ & $1.00-10.00$ \\
\hline sachfremde Belastung & 4 & 2.62 & 1.51 & $1.00-6.75$ & $1.00-10.00$ \\
\hline lernrelevante Belastung & 5 & 5.12 & 2.58 & $1.00-9.00$ & $1.00-10.00$ \\
\hline \multicolumn{6}{|l|}{ Lernerfolg } \\
\hline Wissensabruf & 7 & 0.71 & 0.23 & $0.14-1.00$ & $0.00-1.00$ \\
\hline Wissenstransfer & 14 & 0.77 & 0.18 & $0.36-1.00$ & $0.00-1.00$ \\
\hline
\end{tabular}


Unterschiede zwischen den Versuchsbedingungen auf. Die beiden Modalitäten in der Lernumgebung haben das Blickverhalten nachhaltig beeinflusst. Während die Lernenden in der visuellen Bedingung die visuellen Hinweise zu lesen hatten - bzw. die Hinweise in den Textfeldern mit den Elementen in den Schaltskizzen gegebenenfalls vergleichen mussten, konnten die Lernenden in der auditiven Bedingung während der Hinweisgabe weiterhin die Elemente der Schaltskizzen betrachten. Somit weisen die Unterschiede in den Blickparametern auf substanzielle Effekte beider Modalitäten auf die Aufgabenbearbeitung im Sinne eines Treatment-Checks hin. Allerdings lassen sich die besagten Unterschiede in den Blickparametern nicht direkt auf die sonst damit assoziierten kognitiven Prozesse (z. B. Integration und Organisation) übertragen.

\section{Effekte von auditiven und visuellen Hinweisen (Fragestellung 1)}

Um herauszufinden, ob die Hinweismodalität einen Einfluss auf die kognitive Belastung sowie den Lernerfolg hat, wurde eine multivariate Kovarianzanalyse (MANCOVA) durchgeführt (vgl. Tab. 2). Die Lernenden in der auditiven Bedingung erzielten deskriptiv einen höheren Lernerfolg (Abruf und Transfer), eine geringere inhaltsbedingte und lernrelevante Belastung sowie eine höhere sachfremde Belastung als Lernende in der visuellen Bedingung. Obwohl diese Ergebnisse in eine hypothesenkonforme Richtung weisen, waren sie nicht signifikant. Die Hypothesen 3, 4 und 5 müssen daher zurückgewiesen werden. Als Kontrollvariable hatte Vorwissen einen signifikanten Effekt auf den Lernerfolg und die kognitive

Tabelle 2. Multivariate Kovarianzanalyse zur kognitiven Belastung und zum Lernerfolg getrennt nach Versuchsbedingung mit Vorwissen als Kovariate

\begin{tabular}{|c|c|c|c|c|c|c|c|}
\hline & \multicolumn{2}{|c|}{ Auditiv } & \multicolumn{2}{|c|}{ Visuell } & \multirow[b]{2}{*}{$F(1,43)$} & \multirow[b]{2}{*}{$p$} & \multirow[b]{2}{*}{$\eta_{p}^{2}$} \\
\hline & $M$ & $S D$ & $M$ & $S D$ & & & \\
\hline \multicolumn{8}{|l|}{ Kognitive Belastung } \\
\hline inhaltsbedingte Belastung & 2.35 & 1.34 & 3.25 & 2.17 & 2.47 & .123 & .05 \\
\hline sachfremde Belastung & 2.64 & 1.31 & 2.59 & 1.72 & .01 & .944 & .00 \\
\hline lernrelevante Belastung & 4.41 & 2.78 & 5.83 & 2.19 & 2.78 & .103 & .06 \\
\hline \multicolumn{8}{|l|}{ Lernerfolg } \\
\hline Wissensabruf & 0.76 & 0.26 & 0.66 & 0.19 & 1.34 & .253 & .03 \\
\hline Wissenstransfer & 0.83 & 0.15 & 0.71 & 0.20 & 3.80 & .058 & .08 \\
\hline
\end{tabular}

Tabelle 3. Hierarchisch-multiple Regression zur Vorhersage des Lernerfolgs beim Wissensabruf

\begin{tabular}{|c|c|c|c|c|c|c|}
\hline Variable & $B$ & SEB & $\beta$ & $p$ & $R^{2}$ & $\Delta R^{2}$ \\
\hline Schritt 1 & & & & & .213 & \\
\hline Konstante & .50 & .07 & & & & \\
\hline Vorwissen & .30 & .09 & .46 & .001 & & \\
\hline Schritt 2 & & & & & .236 & .024 \\
\hline Konstante & .62 & .12 & & & & \\
\hline Vorwissen & .28 & .09 & .44 & .002 & & \\
\hline Versuchsbedingung $^{\text {a }}$ & -.07 & .06 & -.16 & .253 & & \\
\hline Schritt $3^{b}$ & & & & & .463 & .227 \\
\hline Konstante & .89 & .12 & & & & \\
\hline Vorwissen & .24 & .08 & .37 & .006 & & \\
\hline Versuchsbedingung ${ }^{a}$ & -.03 & .06 & -.06 & .636 & & \\
\hline inhaltsbedingte Belastung & -.00 & .02 & -.03 & .831 & & \\
\hline sachfremde Belastung & -.05 & .02 & -.31 & .039 & & \\
\hline lernrelevante Belastung & -.03 & .01 & -.37 & .008 & & \\
\hline
\end{tabular}


Belastung $\left(F(5,39)=6.08, p<.001, \eta_{\mathrm{p}}^{2}=.438\right)$. Auf das Lernerfolgsmaß Wissenstransfer zeigte die Analyse zudem einen tendenziellen Vorteil der auditiven Bedingung $(F(1,43)=3.80, p<.100)$.

\section{Prädiktoren für den Wissensabruf und -transfer (Fragestellung 2)}

Um zu prüfen, inwieweit die Hinweismodalität, das Vorwissen und die kognitive Belastung die Unterschiede in den Lernergebnissen beim Wissenabruf und -transfer vorhersagen, wurde jeweils eine hierarchisch-multiple Regression durchgeführt.

Tabelle 3 weist das Vorwissen sowie die extrinsische und lernbezogene Belastung als signifikante Prädiktoren für den Lernerfolg beim Wissensabruf aus. Dies bedeutet, dass ein höherer Lernerfolg beim Wissensabruf festgestellt werden kann je geringer die beiden Belastungsarten ausfallen und je höher das Vorwissen ist. Für die Versuchsbedingung ergab sich kein signifikanter Effekt, da sie kaum zur Varianzaufklärung im Wissensabruf beitrug. Insgesamt erklärten alle eingeschlossenen Variablen 39.6 Prozent der Varianz des Lernerfolgs beim Wissensabruf.

Tabelle 4 weist auf das Vorwissen sowie die extrinsische Belastung als signifikante Prädiktoren für den Lernerfolg beim Wissenstransfer hin. Dies bedeutet, dass ein höherer Lernerfolg beim Wissenstransfer mit einer geringeren wahrgenommenen extrinsischen Belastung und einem höheren Vorwissen einhergeht. Für die Versuchsbedingung ließ sich lediglich eine Tendenz feststellen $(\beta=-.25$, $p<.100)$. Die Bedingung trug wiederum nur einen geringen Teil zur Varianzaufklärung im Wissenstransfer bei. Insgesamt erklärten alle eingeschlossenen Variablen 36.5 Prozent der Varianz des Lernerfolgs beim Wissenstransfer.

Die hierarchisch-multiplen Regressionen weisen auf das Vorwissen sowie die extrinsische Belastung als signifikante Prädiktoren für den Lernerfolg im Wissensabruf als auch im Transfer hin, während die experimentelle Bedingung keinen signifikanten Prädiktor darstellte. Auch wenn die gesamte erklärte Varianz für beide Lernerfolgsmaße ähnlich hoch war, zeigte die hierarchische Analyse einen höheren Einfluss des Vorwissens und der Versuchsbedingung für den Wissenstransfer als für den Wissensabruf. Im Gegensatz dazu halbiert sich in etwa der Erklärungsanteil der Variablen der kognitiven Belastung beim Wissenstransfer. Konkret bedeutet dies, dass Lernende, die während der Lernsitzung eine höhere kognitive Belastung empfanden, ihr Wissen weniger gut abrufen konnten. Beim Transfer war dieser Effekt weniger stark ausgeprägt; hier profitierten die Lernenden primär von ihrem bestehenden Vorwissen. Auf Basis dieser Ergebnisse können die Hypothesen $1 \mathrm{~b}$ und 2 bestätigt werden; Hypothese 1a konnte nur für den Wissensabruf bestätigt werden.

\section{Vorwissensspezifische Blickbewegungen (Fragestellung 3)}

Im Rahmen einer qualitativen Studie wurden die Blickpfade von Lernenden mit niedrigem und hohem Vorwissen

Tabelle 4. Hierarchisch-multiple Regression zur Vorhersage des Lernerfolgs beim Wissenstransfer

\begin{tabular}{|c|c|c|c|c|c|c|}
\hline Variable & $B$ & $S E B$ & $\beta$ & $p$ & $R^{2}$ & $\Delta R^{2}$ \\
\hline Schritt 1 & & & & & .257 & \\
\hline Konstante & .59 & .05 & & & & \\
\hline Vorwissen & .26 & .07 & .51 & .001 & & \\
\hline Schritt 2 & & & & & .317 & .060 \\
\hline Konstante & .74 & .09 & & & & \\
\hline Vorwissen & .24 & .07 & .47 & .001 & & \\
\hline Versuchsbedingung ${ }^{a}$ & -.09 & .05 & -.25 & .058 & & \\
\hline Schritt $3^{b}$ & & & & & .435 & .118 \\
\hline Konstante & .82 & .10 & & & & \\
\hline Vorwissen & .28 & .07 & .55 & .001 & & \\
\hline Versuchsbedingung a & -.09 & .65 & -.25 & .057 & & \\
\hline inhaltsbedingte Belastung & .00 & .02 & .02 & .920 & & \\
\hline sachfremde Belastung & -.04 & .02 & -.36 & .022 & & \\
\hline lernrelevante Belastung & .00 & .01 & .00 & .981 & & \\
\hline
\end{tabular}


hinsichtlich auftretender Regelmäßigkeiten analysiert. Hierzu wurden für beide Bedingungen Extremgruppen hinsichtlich des Vorwissens gebildet und Personen mit weniger als 20 Prozent im Vorwissensscore der Kategorie „niedriges Vorwissen“ und Personen mit voller Punktzahl im Vorwissen der Kategorie „hohes Vorwissen“ zugeordnet. Die Eye-Tracking-Parameter für beide Extremgruppen finden sich im ESM 1 in den Tabellen E8 (globale Parameter) und E9 (lokale Parameter). Die hier dargestellten Blickpfadmuster zeigen charakteristische, innerhalb einer Modalität häufig auftretende, Unterschiede zwischen jeweils zwei Lernenden mit extremen Ausprägungen im Vorwissen (siehe Abb. 2-3). Alle Grafiken beschreiben ein 10-Sekunden-Intervall, das kurz vor der Darbietung des zweiten Hinweises (unten) zur Schaltung (b) beginnt. Zu diesem Zeitpunkt hatten sich die Lernenden bereits in den Kontext der Aufgabe eingefunden, sodass die Blickmuster den Lernprozess nach der anfänglichen Orientierung in der Aufgabe wiedergeben.

Qualitative Ergebnisse der visuellen Bedingung. Abbildung 2 stellt beispielhaft ein Blickmuster bei visueller Hinweisgabe bei einer Person mit niedrigem Vorwissen (links) und bei einer Person mit hohem Vorwissen (rechts) dar. Der Fokus der Person mit niedrigem Vorwissen war überwiegend auf einzelne Elemente innerhalb der angesprochenen oder der benachbarten Schaltung (c) gerichtet: Die Blicke springen sehr oft zwischen dem Text und spezifischen Schaltelementen hin und her; es finden kaum längere Sakkaden statt. Im Gegensatz dazu stellte die Person mit hohem Vorwissen Vergleiche zwischen der aktuell thematisierten Schaltung (b) und den benachbarten Schaltskizzen (a) und (c) an. Erkennbar ist dies an einer höheren Anzahl längerer Sakkaden, die zwischen den Schaltungen stattfinden.
Insgesamt wirkte der visuelle Hinweis bei beiden exemplarisch ausgewählten Personen. Ihre Aufmerksamkeit wurde grundsätzlich auf das im Hinweis angesprochene Element der entsprechenden Schaltung gelenkt. Dies lässt sich an den Sakkaden erkennen, die sich zwischen den in den Hinweisen erwähnten Schaltsymbol (Batterie) und dem Hinweis selbst vollziehen. Darüber hinaus nutzte die Person mit niedrigem Vorwissen jedoch den dargebotenen Hinweis deutlich intensiver als die Personen mit hohem Vorwissen, was sich an der größeren Anzahl von Sakkaden zwischen Hinweis und Schaltung zeigt. Dies könnte daran liegen, dass Lernende mit niedrigerem Vorwissen mehr kognitive Energie investieren mussten, um sich ein mentales Modell der jeweiligen Schaltung aufzubauen.

Qualitative Ergebnisse der auditiven Bedingung. Abbildung 3 stellt beispielhaft ein Blickmuster bei auditiver Hinweisgabe für eine Person mit niedrigem Vorwissen (links) und eine Person mit hohem Vorwissen (rechts) dar. Die Person mit niedrigem Vorwissen fokussierte überwiegend einzelne Elemente innerhalb der angesprochenen Schaltung (b) sowie der beiden benachbarten Schaltungen (a) und (c) und zog Vergleiche zwischen den betrachteten Elementen. Deutlich sind dabei Sprünge (Vergleiche) zwischen den einzelnen Elementen der benachbarten Schaltungen zu erkennen, was sich an den vielen entsprechende Sakkaden zeigt. Im Gegensatz dazu nahm die Person mit hohem Vorwissen während der Hinweisdarbietung neben der angesprochenen Schaltung (b) auch die Schaltungen (c) und (d) sowie die Aufgabenstellung in den Blick. Hier blieben die Sakkaden meist innerhalb einer Schaltung, bevor ein Sprung zur nächsten Schaltung erfolgte. Durch die Sakkaden wird also eine sequenzielle Bearbeitung der Schaltungen erkennbar. Dieses sequentielle „Abarbeiten“ der Schaltungen mit relativ wenigen Elementvergleichen

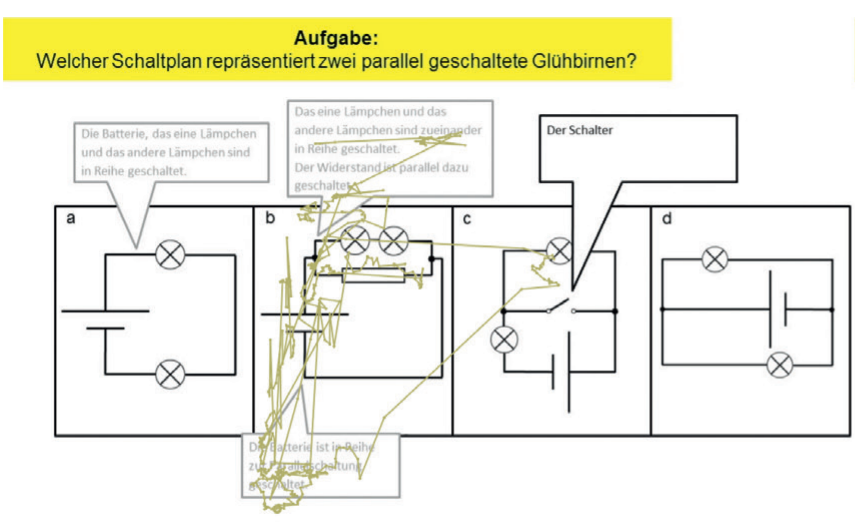

(a)

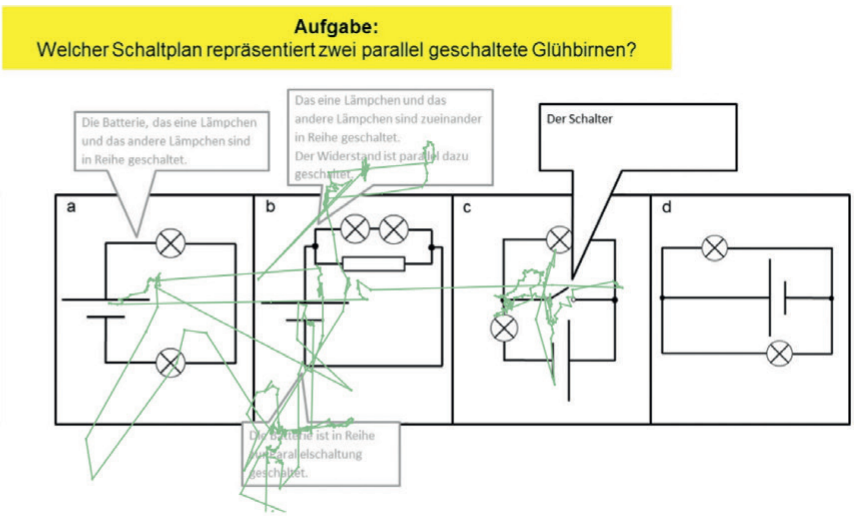

(b)

Abbildung 2. Darstellung von Blickpfadmustern aus der visuellen Bedingung während einer Hinweisgabe. Panel (a) zeigt ein häufiges Blickpfadmuster bei niedrigem Vorwissen (weniger als $20 \%$ im Vorwissensscore). Panel (b) präsentiert ein häufiges Blickmuster bei hohem Vorwissen (100\% im Vorwissensscore). Die Muster stellen aus den beiden 1,5-minütigen Videos jeweils eine 10 Sekunden-Aufzeichnung dar. 


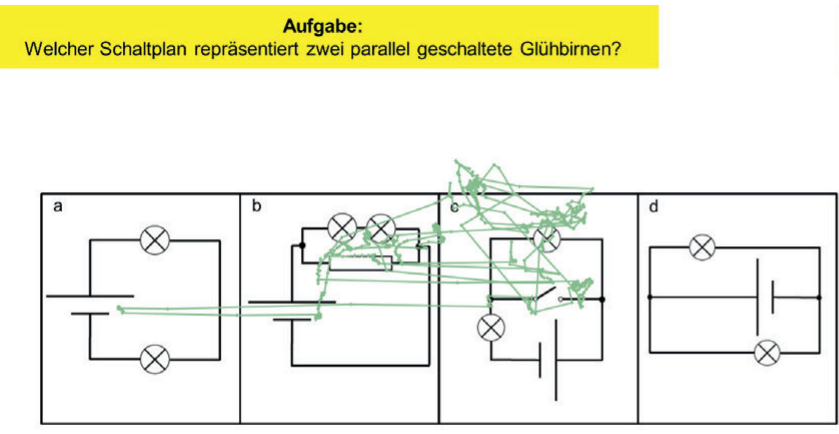

(c)

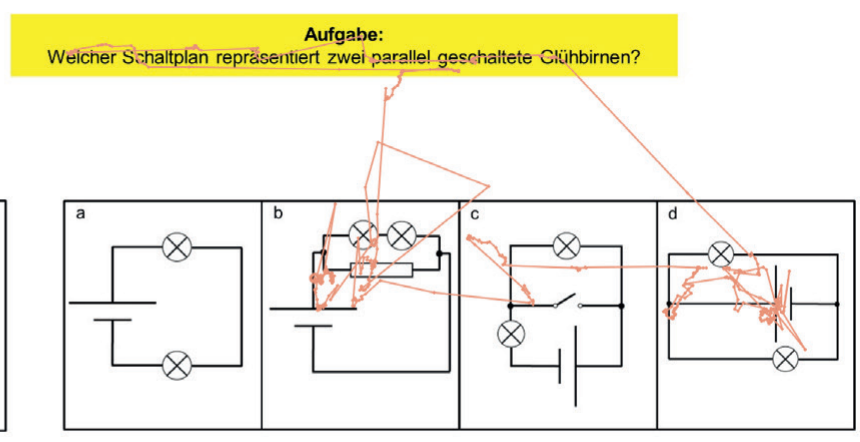

(d)

Abbildung 3. Darstellung von Blickpfadmustern aus der auditiven Bedingung während einer Hinweisgabe. Panel (c) zeigt ein häufiges Blickpfadmuster bei niedrigem Vorwissen (weniger als $20 \%$ im Vorwissensscore). Panel (d) präsentiert ein häufiges Blickmuster bei hohem Vorwissen (100\% im Vorwissensscore). Die Muster stellen aus den beiden 1,5-minütigen Videos jeweils eine 10 Sekunden-Aufzeichnung dar.

könnte dafürsprechen, dass Lernende mit hohem Vorwissen weniger kognitive Energie in den Aufbau eines mentalen Modells der Schaltungen investieren mussten. Insgesamt lenkte der auditive Hinweis jedoch grundsätzlich die Aufmerksamkeit beider Personen auf das im Hinweis erwähnte Element der entsprechenden Schaltung: Beide Lernende wiesen Sakkaden in Richtung des angesprochenen Elements auf.

Zusammenschau der auditiven und visuellen Bedingung. Es zeigte sich, dass in beiden Bedingungen die zwei Personen mit niedrigem Vorwissen ein detail- oder elementorientiertes Blickverhalten aufwiesen: Sie sprangen sehr intensiv zwischen einzelnen Elementen der Schaltungen entweder im Vergleich zum Hinweis oder im Vergleich zur Nachbarschaltung. Diese intensiven Vergleiche könnten als Aufwand zur Erstellung eines mentalen Modells der jeweiligen Schaltung interpretiert werden. Die Blickmuster der beiden Personen mit hohem Vorwissen wiesen deutlich weniger solcher Einzelvergleiche auf, was als ganzheitliches oder schaltungs-orientiertes Blickverhalten charakterisiert werden könnte. Eine mögliche Deutung ist, dass es Personen mit hohem Vorwissen leichter gefallen sein könnte, die jeweiligen Schaltungen zu erfassen. Auch könnte der Aufbau eines mentalen Modells der Schaltungen weniger aufwendig gewesen sein. Für die visuelle Bedingung zeigte sich zudem, dass ein Teil der visuellen Aufmerksamkeit zur Hinweisverarbeitung genutzt wurde, da der Hinweistext von beiden Personen betrachtet wurde (siehe dazu auch die Unterschiede in den lokalen Blickparametern zwischen den Versuchsbedingungen in Tabelle E9 im ESM 1). Insgesamt lenkten sowohl die auditiven als auch die visuellen Hinweise die Aufmerksamkeit aller exemplarisch dargestellten Personen. Im Gegensatz zur visuellen Hinweisgabe war das Blickverhalten der beiden Personen bei auditiver Hinweisgabe jedoch deutlich weniger gelenkt.

\section{Diskussion}

Die vorliegende Studie untersuchte Ergebnisse und individuelle Prozesse des Physik-Lernens mit auditiven und visuellen Hinweisen. Dabei ergaben sich zwischen den Versuchsbedingungen keine statistisch signifikanten Unterschiede in der Wahrnehmung der kognitiven Belastung und im Lernerfolg. Allerdings waren Maße der kognitiven Belastung signifikante Prädiktoren des Lernerfolgs. Zudem zeigten sich bedeutsame Einflüsse des Vorwissens auf Wissensabruf und Wissenstransfer, die sich auch in einem veränderten Blickverhalten widerspiegelten. In beiden Modalitäten wiesen Personen mit niedrigem Vorwissen ein detail- bzw. element-orientiertes Blickmuster und Personen mit hohem Vorwissen ein ganzheitliches bzw. schaltungsorientiertes Blickmuster auf, was wir als Indikatoren für den Aufwand des Erstellens eines mentalen Modells zur Schaltung interpretieren.

\section{Effekte von auditiven und visuellen Hinweisen}

Hinsichtlich der kognitiven Belastung wurde die Annahme überprüft, ob die sachfremde Belastung in der visuellen Bedingung geringer ausfällt als in der auditiven Bedingung (H5). Die Ergebnisse beider Bedingungen waren jedoch annähernd vergleichbar, sodass man vermuten kann, dass die Spezifika beider Bedingungen zu einer vergleichbaren sachfremden Belastung geführt haben. Bei den visuellen Hinweisen könnte es aufgrund der Aufmerksamkeitsteilung zwischen Hinweis und Lernbereich zu einer sachfremden Belastung gekommen sein, was zu einem gewissen Maß durch den Split-Attention-Effekt erklärt werden kann (vgl. Ayres \& Sweller, 2014). Dieser Effekt war bei den auditiven Hinweisen nicht relevant. Allerdings fehlte bei 
den auditiven Hinweisen die repräsentationale Führung (Suthers \& Hundhausen, 2003), was vermutlich eine sachfremde Belastung durch stärkere Transformationsprozesse hervorgerufen hat (Leppink et al., 2015). Diese war erforderlich, um die gehörten Informationen in ein mentales Modell zu überführen. Während die visuellen Hinweise die entsprechenden Referenzen durch die Leitpfeile generierten, mussten diese bei auditiven Hinweisen vom Lernenden selbst mental gebildet werden. Hinzu kommt, dass auditive Hinweise den Fokus der Lernenden nur einmalig leiten und keine Permanenz gewährleisten. Die sich jedoch kaum unterscheidenden Ergebnisse und insgesamt relativ niedrigen Werte der sachfremden Belastung deuten darauf hin, dass beide Hinweismodalitäten die visuelle Aufmerksamkeit vergleichbar leiteten. Auch die Blickmuster beider Bedingungen zeigten einen Fokus der Lernenden auf die relevanten Aspekte im Lernmaterial.

Des Weiteren wurde der Annahme nachgegangen, dass die lernrelevante Belastung in der visuellen Bedingung höher ausfällt als in der auditiven Bedingung (H4), was auf die Möglichkeit des mehrmaligen Wahrnehmens und Verarbeitens der visuellen Hinweise zurückzuführen sein könnte. Die exemplarisch dargestellten Blickmuster dokumentieren zwar, dass die visuellen Hinweise wiederholt verarbeitet wurden, ebenso legen die deskriptiven Werte eine höhere lernrelevante Belastung in der visuellen Bedingung nahe - ein signifikanter Unterschied zu Gunsten der visuellen Bedingung ergab sich jedoch nicht. Die deskriptiv geringere lernbezogene Belastung in der auditiven Bedingung deutet wiederum auf einen Modalitätseffekt hin. Dieser teilt die Informationslast auf zwei Sinneskanäle auf, wodurch mehr Ressourcen für die Organisation und Integration der Informationen zur Verfügung stehen (Low \& Sweller, 2014). Dies lässt sich empirisch durch die vorliegende Untersuchung allerdings nicht bestätigen. Insgesamt deuten die Ergebnisse für den Bereich der kognitiven Belastung darauf hin, dass für jede Hinweismodalität spezifische Aspekte zur kognitiven Belastung beitragen.

In Bezug auf den Lernerfolg wurde die Annahme eines modalitätsspezifischen Unterschieds überprüft. Basierend auf dem Modalitätseffekt (Low \& Sweller, 2014) hätte sich ein erhöhter Wissensabruf und Wissenstransfer in der auditiven Bedingung ergeben sollen (H3). Vielmehr zeigte sich, dass in beiden Versuchsbedingungen nahezu vergleichbar gelernt wurde. Hierzu lassen sich Gründe anführen, die für die Effektivität beider Hinweismodalitäten sprechen könnten:

Die Effektivität visueller Hinweise könnte durch die Möglichkeit der wiederholten Hinweisbetrachtung bedingt sein, da Lernende bei Bedarf die visuellen Darbietungen mehrmals wahrnehmen können, sowie durch die repräsentationale Führung ausgehend von den visuellen Hinweisen (Suthers \& Hundhausen, 2003). Dies zeigte sich auch in der qualitativen Analyse der beiden exemplarisch ausgewählten Blickmuster, bei denen sich jeweils mehrere Sakkaden zwischen dem dargebotenen Hinweis und dem erwähnten Schaltsymbol vollzogen.

Die positive Tendenz auditiver Hinweise zu visuellen Repräsentationen könnte durch die simultane Verarbeitung der Informationen erklärt werden. Insbesondere in videobasierten Lernumgebungen, bei denen der Lernprozess durch die Systemsteuerung an eine Zeitvorgabe gebunden ist, könnten sich auditive Hinweise als lernförderlich erweisen. Im Gegensatz zu visuellen Hinweisen könnte bei ihnen mehr freie Zeit zur kognitiven Verarbeitung der Schaltskizzen zur Verfügung gestanden haben, da die auditiv dargebotenen Hinweise nicht gelesen werden mussten, wenn sie mit den piktoralen Darstellungen in Beziehung gesetzt wurden. Betrachtet man die auditiven und visuellen Blickmuster im Vergleich, so könnte das weniger gelenkte Blickverhalten in der auditiven Bedingung auf das Vorhandensein von mehr freier Verarbeitungszeit hindeuten. Diese Vermutung spiegelt sich auch in den substantiellen Veränderungen der Blickbewegungen durch die Wirkung des Treatments wider (vgl. hierzu Tabelle E6 im ESM 1). Es zeigte sich, dass der Fokus der Lernenden in der auditiven Bedingung länger und häufiger auf den relevanten Bereichen (elektrische Schaltskizzen) lag.

\section{Prädiktoren für den Wissensabruf und -transfer}

Im Hinblick auf verlässliche Prädiktoren zur Vorhersage der Unterschiede in den Lernergebnissen wurden das Vorwissen, die Hinweismodalität und die drei Indikatoren der kognitiven Belastung untersucht.

Das Vorwissen erwies sich in beiden Lernerfolgsarten durchgehend als stärkster Prädiktor (H2). Wie bereits aus anderen Arbeiten zum Vorwissen (z.B. Ertl et al., 2005; Hattie, 2009) bekannt ist, belegt auch der vorliegende Befund, dass fachspezifische Vorkenntnisse grundsätzlich positiv auf den gesamten Lernerfolg wirken. Es ist festzuhalten, dass das Vorwissen als eine bedeutsame Voraussetzung zum erfolgreichen Lösen domänenspezifischer Aufgabenstellungen komplexer Art in hinweisbegleiteten Lernumgebungen anzusehen ist. Adaptive Lernumgebungen (siehe Leutner, 2002) könnten in solchen Kontexten Vorwissensunterschiede abmildern, indem sie vorwissensschwächeren Lernenden elaboriertere Erklärungen und mehr Lernzeit zugestehen.

Die sachfremde Belastung erwies sich in beiden Lernerfolgsarten als ein verlässlicher Prädiktor für die Unterschiede in den Lernergebnissen (H1b). Der signifikante Effekt zeigt, dass Lernende, welche die Hinweise als nützlich wahrnahmen, insgesamt einen höheren Lernerfolg 
erzielten. Das Ergebnis steht im Einklang mit der Theorie der kognitiven Belastung (Sweller et al., 1998), die bei geringerer sachfremder Belastung einen höheren Lernerfolg annimmt. Zudem kann dieser Befund als weitere empirische Evidenz für die intendierte Nützlichkeit der Hinweisgabe in multimedialen Lernumgebungen angesehen werden.

Die lernrelevante Belastung erwies sich lediglich beim Wissensabruf als prädiktiv (H1a). Dieser signifikante Effekt zeigt, dass Lernende, welche die hinweisbegleiteten Videos als verständnisverbessernd ansahen, einen höheren Lernerfolg im Wissensabruf erzielten. Dies deutet im Vergleich zum Ergebnis des Wissenstransfers darauf hin, dass die hinweisbegleitete Lernumgebung ein eher oberflächliches Verständnis förderte. Vermutlich konnte aufgrund der begrenzten Bearbeitungsdauern der Aufgaben keine eingehende Auseinandersetzung mit dem Lerngegenstand stattfinden, sodass diese Voraussetzung für einen erfolgreichen Transfer des Wissens fehlte.

Generell ist festzustellen, dass sich der Einfluss der kognitiven Belastung beim Transfer im Vergleich zum Wissensabruf halbierte, was auf den stärkeren Einfluss der kognitiven Belastung in der Lernsitzung auf den späteren Wissensabruf hinweist. Im Gegensatz dazu profitierten beim Transfer primär Lernende, die schon vor der Lerneinheit ein hohes Vorwissen besaßen. Dies kann bedeuten, dass für den Wissenstransfer individuelle Lernvoraussetzungen die Effekte einer Lerneinheit moderieren, während beim Wissensabruf die Effekte der Lernumgebung im Vordergrund stehen.

\section{Vorwissensspezifische Blickbewegungen}

Die Unterschiede im Blickverhalten zwischen den jeweils beiden exemplarisch ausgewählten Personen mit hohem und niedrigem Vorwissen innerhalb der beiden Modalitäten könnten auf ihre unterschiedlichen mentalen Belastungen zurückzuführen sein (siehe dazu auch Tabelle E7 im ESM 1). Für die Personen mit niedrigem Vorwissen ergab sich nach der Theorie der kognitiven Belastung (Sweller et al., 1998) insbesondere in einer Lernumgebung mit starker Elementinteraktivität vermutlich eine höhere inhaltsbezogene Belastung (Leppink et al., 2015). Durch den Verstehens- und Verarbeitungsprozess der teilweise noch unbekannten Zusammenhänge und Schaltsymbole könnte sich auch ihre lernbezogene Belastung erhöht haben. Zudem dürfte die eingehendere Betrachtung des visuellen Hinweises in der visuellen Bedingung bei der Person mit niedrigem Vorwissen weitere kognitive Ressourcen intrinsischer und lernbezogener Art gebunden haben. Ein möglicher Grund hierfür könnte im Prozess des Chunkings (vgl. Miller, 1956) liegen. Demnach könnte das Zusammenfassen von Einzelelementen im Sinne des Chunkings bei Personen mit hohem Vorwissen leichter vonstatten gegangen sein. Beispielsweise könnte das Symbol für „Lampe“ mit der Bedeutung für Lampe bereits einen Chunk gebildet haben, sodass die dazu notwendigen Verknüpfungs- und Vergleichsprozesse nicht mehr stattfinden mussten und dadurch mentale Kapazität verfügbar war. Wohingegen Personen mit niedrigem Vorwissen vermutlich solche Vergleiche und Verknüpfungen explizit durchführen mussten, was einen Teil der kognitiven Kapazität verbrauchte. Somit könnte auf Basis der Theorie nach Sweller et al. (1998) vermutet werden, dass während des hinweisbegleiteten Lernprozesses für Vergleiche der thematisierten Schaltung mit anderen Schaltungen kaum weitere mentale Ressourcen übrig blieben, weshalb der Fokus überwiegend auf den einzelnen Elementen und auf deren Vergleich untereinander lag. Insgesamt bestätigen die Blickbewegungen sowie die damit assoziierten Verarbeitungsprozesse aus der qualitativen Studie die Differenzierung in vorwissensstarke und vorwissensschwache Lernende aus dem Vorwissenstest.

Betrachtet man die qualitativen Ergebnisse einmal unabhängig vom Vorwissen, so lässt sich in der auditiven Bedingung ein weniger geleitetes Blickverhalten vorfinden. Dies lässt sich möglicherweise darauf zurückführen, dass die auditive Bedingung grundsätzlich keine repräsentationale Führung aufweist, welche die Aufmerksamkeit der Lernenden prinzipiell stärker auf die einzelnen Elemente der Schaltungen lenken sollte. Demgegenüber ist zu verzeichnen, dass sich die beiden Personen in der auditiven Bedingung wahrscheinlich intensiver mit den dargebotenen Schaltungen auseinandersetzen konnten, was die Darbietung von Hinweisen in der visuellen Bedingung vermutlich generell erschwerte (vgl. hierzu auch die Unterschiede in den lokalen Blickparametern in Tabelle E6 im ESM 1).

\section{Folgerungen}

Obwohl sich eine Tendenz für bessere Lernergebnisse durch die auditive Hinweisgabe abzeichnete, hat die vorliegende Studie gezeigt, dass in einer komplexen physikalischen Lernumgebung beide Hinweismodalitäten nützlich sein können. Während Lernenden durch die auditiven Hinweise vermutlich mehr Verarbeitungszeit für den Vergleich der Schaltungen zur Verfügung stand, profitierten Lernende möglicherweise von der repräsentationalen Führung der visuellen Hinweise. Wie in vielen pädagogischen Settings bildete das Vorwissen den stärksten Prädiktor des Lernerfolgs. Zudem deuten die vorwissensspezifischen Blickmuster auf ein eher ganzheitliches oder schaltungs-orientiertes Blickverhalten Lernender mit hohem Vorwissen hin, die ein elaborierteres Verständnis 
widerspiegeln könnten. Im Rahmen einer Adaption von Instruktionszeit und -sequenz nach Leutner (2002) ergeben sich als Konsequenzen für zukünftige Lernumgebungen: Lernenden mit niedrigem Vorwissen sollte mehr Lernzeit zugestanden und eine spezifische Instruktion, die diese schaltungsorientierte Aufgabenbearbeitung anleitet, gegeben werden.

\section{Limitationen}

Wie jedes Lernexperiment weist auch diese Untersuchung Einschränkungen auf. Im Hinblick auf einige Ergebnisse, die das Signifikanzniveau von fünf Prozent nicht erreichten, muss man festhalten, dass der Umfang der Stichprobengröße leider nur eine geringe Teststärke bietet. Die Generierung von Daten im Zusammenhang mit Eye-Tracking erweist sich generell als aufwendig, da aus technischen Gründen nur sequenzielle Einzeltestungen möglich sind. Zusätzlich ist zu beachten, dass bei Eye-TrackingStudien eine relativ hohe Variabilität in den Blickparametern zu verzeichnen ist. Wenn die Daten zudem nach Modalität und Vorwissen aufgespalten werden, verkleinert sich die Probandenanzahl in den Bedingungen deutlich. Gegenfurtner et al. (2011) konstatierten in ihrer Metaanalyse, dass zu kleine Stichproben in Studien, die den $\mathrm{Zu}-$ sammenhang von Vorwissensunterschieden und Blickbewegungen betrachten, zu einer Uneinheitlichkeit der wissenschaftlichen Befundlage beitragen.

Auch ist die Reliabilität des Tests zum Wissensabruf nicht im optimalen Bereich. Hierbei ist jedoch zu beachten, dass es sich bei den eingesetzten Items um eine Wiedergabe der Aufgaben aus der Lernphase handelte. Diese können also in der Lernphase erworbenes Wissen sehr genau reproduzieren - allerdings auch durch reines Erinnern gelöst werden. Stadler, Sailer und Fischer (2021) argumentieren, dass Wissen ein formatives Konstrukt darstellt, welches sich aus unterschiedlichen Aspekten zusammensetzt. Sie fordern stärker auf ein optimales Abdecken der theoretischen Breite des Wissenskonstrukts $\mathrm{zu}$ achten als auf eine Maximierung der Interitemkorrelationen. Daher wurden die Items in ihrer vollen Breite beibehalten anstatt durch ein Weglassen von Items zu versuchen, ein höheres Alpha zu erreichen.

Die Ergebnisinterpretation wird zudem dadurch eingeschränkt, dass die Hinweisgabe im Experiment eine inhaltsspezifische Unterstützung mit einer Referenz auf die jeweilige Schaltung kombinierte und diese mit der Modalität verbunden war. Diese Designentscheidung orientierte sich an der ökologischen Validität des Settings. Die beiden Settings bestanden aus authentischen Lernmaterialien, die in der visuellen Bedingung eine Animation und in der auditiven Bedingung eine vertonte Grafik enthielten. Da in einem alternativen $2 \times 2$-Design, in dem sowohl die inhaltsspezifische Unterstützung als auch die entsprechenden Referenzen jeweils, entweder auditiv oder visuell dargeboten worden wären, hätte eine der Bedingungen visuelle inhaltsspezifische Hinweise und auditive Referenzen enthalten. Die Lernenden hätten also einen Text lesen müssen, während der auditive Kanal die Schaltung referenziert hätte - ein Setting das wenig sinnvoll erscheint. Eine auditive Bedingung mit visuellen Hinweisreizen wäre zwar möglich gewesen - allerdings wären die hier zu erwartenden Effekte trivial gewesen und wurden in anderen Kontexten bereits eingehend untersucht.

Generell ist die lernförderliche Wirkung von Hinweisen bekannt (z.B. Kirschner et al., 2006; Richter et al., 2016; Schneider et al., 2018). Daher stand im vornehmlichen Interesse der Forschung, welche Hinweismodalität sich bei einer komplexen physikalischen Lernumgebung als effektiver erweist und nicht, ob Hinweise überhaupt lernwirksam sind. Die damit verbundene Frage nach einer Kontrollgruppe ohne instruktionale Hinweise gilt spätestens seit den vielzitierten Veröffentlichungen von Mayer (2004) sowie Kirschner et al. (2006) als wenig sinnvoll.

\section{Implikationen und Ausblick}

Zusammenfassend lässt sich festhalten, dass sowohl auditive als auch visuelle Hinweise als Maßnahme zur instruktionalen Unterstützung in videobasierten Lernumgebungen in Erwägung gezogen werden können. Im naturwissenschaftlichen Bereich konzentrieren sich viele Studien zu visuellen Repräsentationen auf Mittelwertvergleiche von Eye-Tracking Parametern (z.B. Klein et al., 2020; Saß et al., 2017). Dadurch geraten meist individuelle Unterschiede zwischen den Teilnehmenden oder auch Unterschiede zwischen Subgruppen der Teilnehmenden aus dem Blick. Abhilfe schaffen qualitative Analysen der Blickmuster, da sie differenziertere Erkenntnisse über den Bearbeitungsprozess von dargebotenen Informationen aus komplexen Darstellungen ermöglichen. Perspektivisch könnten solche Blickmuster genutzt werden, um adaptiv Hilfen in Form von Hinweisen einzublenden. Jedoch ist es wichtig, die qualitativen Ergebnisse zu systematisieren und quantitativ zu untersuchen, damit sie als Beitrag zur domänenspezifischen Forschung zu visuellen Repräsentationen mit Blickbewegungsmessungen stärker generalisierbar sind.

Eine Kernerkenntnis der Studie ist, dass beide Bedingungen - auch wenn sie sich deutlich in der Umsetzung und den quantitativen Blickparametern unterscheiden doch ähnliche Prozesse bei Lernenden mit niedrigem und Lernenden mit hohem Vorwissen unterstützt haben. Zukünftige Studien sollten daher - jenseits der Effekte auf 
die Lernerfolge - untersuchen, wie die jeweiligen Hinweise spezifische Aspekte und Prozesse unterstützen können. Dadurch könnte die Hinweismodalität besser auf das verfolgte Lernziel abgestimmt werden, um die lernförderliche Wirkung einer Lernumgebung zu erhöhen. Gerade in den Naturwissenschaften könnte es beispielsweise sinnvoll sein, Fachbegriffe durch visuell präsentierte Textfelder zu vermitteln (vgl. Watzka et al., 2021). Gleichzeitig scheinen aber für andere Lernziele, wie etwa das Vergleichen verschiedener Schaltungen, auditive Hinweise besser geeignet zu sein. Hier könnten speziell qualitative Analysen von Blickmustern in den naturwissenschaftlichen Domänen auch dort verbreitete Lernschwierigkeiten aufdecken.

In Bezug auf die vorwissensabhängigen Unterschiede in den Blickmustern und die Komplexität des Lerngegenstandes erscheinen sowohl eine zeitliche als auch eine instruktionale Adaptierbarkeit der Lernumgebung (vgl. Leutner, 2002) für Lernende mit wenig Vorwissen in beiden Hinweismodalitäten sinnvoll: Durch eine individuell angepasste Lernzeit könnten die dargebotenen Informationen eingehender verarbeitet und miteinander in Beziehung gesetzt werden. In der auditiven Bedingung wäre zusätzlich die Funktion der individuellen Wiederholbarkeit auditiver Hinweise hilfreich, um nicht vollständig erfasste Informationen gänzlich aufnehmen zu können. Auch Bedingungen systematisch zu wechseln, könnte die Wirkung der Hinweisgabe noch weiter verstärken. Studien dazu sollten zukünftig nicht nur die Lernergebnisse, sondern im Sinne eines Mixed-Method-Ansatzes auch Lernund Bearbeitungsprozesse differenziert untersuchen.

\section{Elektronisches Supplement (ESM)}

Das elektronische Supplement ist mit der Online-Version dieses Artikels verfügbar unter https://doi.org/10.1024/ 1010-0652/a000331

ESM 1. Tabellen E5-E9 und Abbildungen E4 und E5 $(\mathrm{PDF})$

\section{Literatur}

Alemdag, E. \& Cagiltay, K. (2018). A systematic review of eye tracking research on multimedia learning. Computers \& Education, 125, 413 - 428. https://doi.org/10.1016/j.compedu.2018.06.023 Ayres, P. \& Sweller, J. (2014). The split-attention principle in multimedia learning. In R. E. Mayer (Ed.), The Cambridge handbook of multimedia learning ( $2^{\text {nd }}$ ed., pp. $206-246$ ). New York: Cambridge University Press.
Brünken, R., Seufert, T. \& Zander, S. (2005). Förderung der Kohärenzbildung beim Lernen mit multiplen Repräsentationen. Zeitschrift für Pädagogische Psychologie, 19(1/2), 61 -75. https:// doi.org/10.1024/1010-0652.19.12.61

Butcher, K. (2006). Learning from text with diagrams: Promoting mental model development and inference generation. Journal of Educational Psychology, 98(1), 182-197. https://doi.org/ 10.1037/0022-0663.98.1.182

Cook, M. P. (2006). Visual representations in science education: The influence of prior knowledge and cognitive load theory on instructional design principles. Science Education, 90, 1073 -1091. https://doi.org/10.1002/sce.20164

Crooks, S. M., Cheon, J., Inan, F., Ari, F. \& Flores, R. (2012). Modality and cueing in multimedia learning: Examining cognitive and perceptual explanations for the modality effect. Computers in Human Behavior, 28, 1063-1071. https://doi.org/10.1016/j. chb.2012.01.010

De Koning, B. B., Tabbers, H. K., Rikers, R. M. J. P. \& Paas, F. (2009) Towards a framework for attention cueing in instructional animations: Guidelines for research and design. Educational Psychology Review, 21,113-140. https://doi.org/10.1007/s10648009-9098-7

De Koning, B. B., Tabbers, H. K., Rikers, R. M. J. P. \& Paas, F. (2010). Attention guidance in learning from a complex animation. Seeing is understanding? Learning and Instruction, 20, 111-122. https:// doi.org/10.1016/j.learninstruc.2009.02.010

Deutsche Gesellschaft für Psychologie (Hrsg.). (2018). Ethisches Handeln in der psychologischen Forschung: Empfehlungen der Deutschen Gesellschaft für Psychologie für Forschende und Ethikkommissionen. Göttingen: Hogrefe.

Engelhardt, P. V. \& Beichner, R. J. (2004). Students' understanding of direct current resistive electrical circuits. American Journal of Physics, 72(1), 98 -115. https://doi.org/10.1119/1.1614813

Ertl, B., Fischer, F. \& Mandl, H. (2006). Conceptual and socio-cognitive support for collaborative learning in videoconferencing environments. Computers \& Education, 47(3), 289-315. https:// doi.org/10.1016/j.compedu.2004.11.001

Ertl, B., Kopp, B. \& Mandl, H. (2005). Effects of an individual's prior knowledge on collaborative knowledge construction and individual learning outcomes in videoconferencing. In T. Koschmann, D. Suthers \& C. Chan (Eds.), Computer supported collaborative learning 2005: the next 10 years! (pp.145-154). Mahwah, NJ: Lawrence Erlbaum Associates.

Ertl, B., Kopp, B. \& Mandl, H. (2008). Supporting learning using external representations. Computers \& Education, 51(4), 1599 - 1608. https://doi.org/10.1016/j.compedu.2008.03.001

Gegenfurtner, A., Lethinen, E. \& Säljö, R. (2011). Expertise differences in the comprehension of visualizations: A meta-analysis of eye-tracking research in professional domains. Educational Psychology Review, 23, 523-552. https://doi.org/10.1007/ s10648-011-9174-7

Glaser, M. \& Schwan, S. (2015). Explaining pictures: how verbal cues influence processing of pictorial learning material. Journal of Educational Psychology, 16, 1006-1018. https://doi. org/10.1037/edu0000044

Glaser, M. \& Schwan, S. (2020). Combining verbal and visual cueing: Fostering learning pictorial content by coordinating verbal explanations with different types of visual cueing. Instructional Science, 48,159-182. https://doi.org/10.1007/s11251-020-09506-5

Hattie, J. (2009). Visible learning. A synthesis of over 800 metaanalysis relating to achievement. New York: Routledge.

Hegarty, M., Carpenter, P. A. \& Just, M. A. (1991). Diagrams in the comprehension of scientific texts. In R. Barr, M. L. Kamil, P. B. Mosenthal \& P. D. Pearson (Eds.), Handbook of reading research (Vol. 2, pp.641 - 668). Mahwah, NJ: Lawrence Erlbaum Associates. 
Holmqvist, K., Nyström, M., Andersson, R., Dewhurst, R., Jarodzka, H. \& van de Weijer, J. (2011). Eye tracking. A comprehensive guide to methods and measures. Oxford: University Press.

Huk, T., Steinke, M. \& Floto, C. (2010). The educational value of visual cues and 3D representational format in a computer animation under restricted and realistic conditions. Instructional Science, 38, 455-469. https://doi.org/10.1007/s11251-009-911 6-7

Hyönä, J. (2010). The use of eye movements in the study of multimedia learning. Learning and Instruction, 20, 172-176. https:// doi.org/10.1016/j.learninstruc. 2009.02.013

Kalyuga, S. (2012). Instructional benefits of spoken words: A review of cognitive load factors. Educational Research Review, 7, 145 - 159. https://doi.org/10.1016/j.edurev.2011.12.002

Kalyuga, S., Chandler, P. \& Sweller, J. (2000). Incorporating learner experience into the design of multimedia instruction. Journal of Educational Psychology, 92(1), 126 -136. https://psycnet.apa. org/doi/10.1037/0022-0663.92.1.126

Kekule, M. (2014). Students' approaches when dealing with kinematics graphs explored by eye-tracking research method. Proceedings of the Frontiers in Mathematics and Science Education Research Conference, 108-117.

Kirschner, P., Sweller, J. \& Clark, R. E. (2006). Why minimal guidance during instruction does not work: an analysis of the failure of constructivist, discovery, problem-based, experimental, and inquiry-based teaching. Educational Psychologist, 41(2), 75-86. https://doi.org/10.1207/s15326985ep4102_1

Klein, P., Becker, S., Küchemann, S. \& Kuhn, J. (2021). Test of understanding graphs in kinematics: Item objectives confirmed by clustering eye movement transitions. Physical Review Physics Education Research, 17(1). https://doi.org/10.1103/PhysRev PhysEducRes.17.013102

Klein, P., Lichtenberger, A., Küchemann, S., Becker, S., Kekule, M., Viiri, J., Baadte, C., Vaterlaus, A. \& Kuhn, J. (2020). Visual attention while solving the test of understanding graphs in kinematics: an eye-tracking analysis. European Journal of Physics, 41(2). https://doi.org/10.1088/1361-6404/ab5f51

Klein, P., Viiri, J. \& Kuhn, J. (2019). Visual cues improve students' understanding of divergence and curl: Evidence from eye movements during reading and problem solving. Physical Review Physics Education Research, 15(1). https://doi.org/10.1103/ PhysRevPhysEducRes.15.010126

Klein, P., Viiri, J., Mozaffari Chanijani, S. S., Dengel, A. \& Kuhn, J. (2018). Instruction-based clinical eye-tracking study on the visual interpretation of divergence: How do students look at vector field plots? Physical Review Physics Education Research, 14(1). https://doi.org/10.1103/PhysRevPhysEducRes.14.010116

Krejtz, K., Duchowski, A. T., Krejtz, I., Kopacz, A. \& ChrząstowskiWachtel, P. (2016). Gaze transition when learning with multimedia. Journal of Eye Movement Research, 9(1), 1-17. https://doi. org/10.16910/jemr.9.1.5

Kriz, S. \& Hegarty, M. (2007). Top-down and bottom-up influences on learning from animations. International Journal of HumanComputer Studies, 65, 911 -930. https://dx.doi.org/10.1016/j. ijhcs.2007.06.005

Kühl, T., Scheiter, K., Gerjets, P. \& Edelmann, J. (2011). The influence of text modality on learning with static and dynamic visualizations. Computers in Human Behavior, 27(1), 29 - 35. https:// psycnet.apa.org/doi/10.1016/j.chb.2010.05.008

Leppink, J., Pass, F., van der Vleuten, C. P. M., van Gog, T. \& van Merriënboer, J.J. G. (2013). Development of an instrument for measuring different types of cognitive load. Behavior Research Methods, 45, 1058-1072. https://doi.org/10.3758/s13428-0130334-1

Leppink, J., Pass, F., van der Vleuten, C. P. M., van Gog, T. \& van Merriënboer, J. J. G. (2014). Effects of pairs of problems and exam- ples on task performance and different types of cognitive load. Learning and Instruction, 30, 32 - 42. http://doi.org/10.1016/j. learninstruc.2013.12.001

Leppink, J., van Gog, T., Paas, F. \& Sweller, J. (2015). Cognitive load theory: researching and planning teaching to maximise learning. In J. Cleland \& S. J. Durning (Eds.), Researching Medical Education (pp. 207 - 218). Chichester, UK: Wiley \& Blackwell.

Leutner, D. (2002). Adaptivität und Adaptierbarkeit multimedialer Lehr- und Informationssysteme. In L.J. Issing \& P. Klimsa (Hrsg.), Information und Lernen mit Multimedia und Internet: Lehrbuch für Studium und Praxis (S.114-125). Weinheim: Beltz PVU.

Leutner, D., Opfermann, M. \& Schmeck, A. (2014). Lernen mit Medien. In T. Seidel \& A. Krapp (Hrsg.), Pädagogische Psychologie (S. 297 - 322). Weinheim: Beltz.

Lindner, M. A., Eitel, A., Thoma, G.-B., Dalehefte, I. M., Ihme, J. M. \& Köller, O. (2014). Tracking the decision-making process in multiple-choice assessment: Evidence from eye movements. Applied Cognitive Psychology, 28(5), 738 - 752. https://doi.org/10.1002/ acp. 3060

Low, R. \& Sweller, J. (2014). The modality principle in multimedia learning. In R. E. Mayer (Ed.), The Cambridge handbook of multimedia learning ( $2^{\text {nd }}$ ed., pp. $227-246$ ). New York, NY: Cambridge University Press. https://doi.org/10.1017/CB09781139547369.012

Mayer, R. E. (2004). Should there be a three-strikes rule against pure discovery learning? The case for guided methods of instruction. American Psychologist, 59(1), 14-19. https://doi. org/10.1037/0003-066x.59.1.14

Mayer, R. E. (Ed.). (2014). Cognitive theory of multimedia learning. The Cambridge handbook of multimedia learning ( $2^{\text {nd }}$ ed., pp. 43 -71). New York, NY: Cambridge University Press. https:// doi.org/10.1017/CB09781139547369.005

Mayer, R. E. \& Moreno, R. (1998). A split-attention effect in multimedia learning: Evidence for dual processing systems in working memory. Journal of Educational Psychology, 90(2), 312 - 320. https://psycnet.apa.org/doi/10.1037/0022-0663.90.2.312

Miller, G. (1956). The magic number seven, plus or minus two: Some limits on our capacity for processing information. Psychological Review, 63(2), 81 - 97. https:///doi.org/10.1037/h0043 158

Mozaffari Chanijani, S. S., Klein, P., Bukhari, S. S., Kuhn, J. \& Dengel, A. (2016). Entropy based transition analysis of eye movement on physics representational competence. UbiComp'16: Proceedings of the 2016 ACM International Joint Conference on Pervasive and Ubiquitous Computing: Adjunct, 1027 - 1034. https:// doi.org/10.1145/2968219.2968564

Raney, G. E., Campbell, S. J. \& Bovee, J. C. (2014). Using eye movements to evaluate the cognitive processes involved in text comprehension. Journal of Visualized Experiments, 83. https://doi. org/10.3791\%2F50780

Richter, J., Scheiter, K. \& Eitel, A. (2016). Signaling text-picture relations in multimedia learning: A comprehensive meta-analysis. Educational Research Review, 17, 19-36. https://doi. org/10.1016/j.edurev.2015.12.003

Rummer, R., Fürstenberg, A. \& Schweppe, J. (2008). Lernen mit Texten und Bildern. Der Anteil akustisch-sensorischer Information am Zustandekommen des Modalitätseffekts. Zeitschrift für Pädagogische Psychologie, 22(1), 37 - 45. https://doi.org/10. 1024/1010-0652.22.1.37

Saß, S., Schütte, K. \& Lindner, M. A. (2017). Test-takers' eye movements: Effects of integration aids and types of graphical representations. Computers \& Education, 109, 85 -97. https://doi. org/10.1016/j.compedu.2017.02.007

Scheiter, K. \& Eitel, A. (2015). Signals foster multimedia learning by supporting integration of highlighted text and diagram elements. Learning and Instruction, 36, 11-26. https://doi. org/10.1016/j.learninstruc.2014.11.002 
Schüler, A. (2017). Investigating gaze behavior during processing of inconsistent text-picture information: Evidence for text-picture integration. Learning and Instruction, 49, 218-231. https://doi. org/10.1016/j.learninstruc. 2017.03.001

Seufert, T. \& Brünken, R. (2006). Cognitive load and the format of instructional aids for coherence formation. Applied Cognitive Psychology, 20, 321 - 331. https://doi.org/10.1002/acp.1248

Seufert, T., Schütze, M. \& Brünken, R. (2009). Memory characteristics and modality in multimedia learning: An aptitude-treatment-interaction-study. Learning and Instruction, 19(1), 28 - 42. https://doi.org/10.1016/j.learninstruc.2008.01.002

Schneider, S., Beege, M., Nebel, S. \& Rey, G. D. (2018). A meta-analysis of how signaling affects learning with media. Educational Research Review, 23, 1-24. https://doi.org/10.1016/j.edurev. 2017.11.001

Song, M. \& Bruning, R. (2016). Exploring effects of background context familiarity and signaling on comprehension, recall, and cognitive load. Educational Psychology, 36(4), 691 - 718. https:// doi.org/10.1080/01443410.2015.1072133

Stadler, M., Sailer, M. \& Fischer, F. (2021). Knowledge as a formative construct: A good alpha is not always better. New Ideas in Psychology, 60. https://doi.org/10.1016/j.newideapsych.2020.100 832

Suthers, D. D. \& Hundhausen, C. D. (2003). An experimental study of the effects of representational guidance on collaborative learning processes. The Journal of the Learning Sciences, 12(2), 183 - 218. https://doi.org/10.1207/S15327809JLS1202_2

Sweller, J., van Merriënboer, J. J. G. \& Paas, F. G. (1998). Cognitive architecture and instructional design. Educational Psychology Review, 10(3), $251-296$.

Tabbers, H. K., Martens, R. L. \& van Merriënboer, J.J. G. (2004). Multimedia instructions and cognitive load theory: Effects of modality and cueing. British Journal of Educational Psychology, 74(1), 71 -81. https://doi.org/10.1348/000709904322848824

Tindall-Ford, S., Chandler, P. \& Sweller, J. (1997). When two sensory modes are better than one. Journal of Experimental Psychology: Applied, 3(4), $257-287$. https://psycnet.apa.org/doi/10.1037/ $1076-898 \times .3 .4 .257$

van Gog, T. (2014). The signaling (or cueing) principle in multimedia learning. In R. E. Mayer (Ed.), The Cambridge handbook of multimedia learning ( $2^{\text {nd }}$ ed., pp. 263 - 278). New York, NY: Cambridge University Press.

van Gog, T. \& Jarodzka, H. (2013). Eye tracking as a tool to study and enhance cognitive and metacognitive processes in computer-based learning environments. In R. Azevedo \& V. Aleven (Eds.), International handbook of metacognition and learning technologies (pp. $143-156)$. New York: Springer US.

Wang, X., Lin, L., Han, M. \& Spector, J. M. (2020). Impacts of cues on learning: Using eye-tracking technologies to examine the functions and designs of added cues in short instructional videos. Computers in Human Behavior, 107. https://doi.org/10.1016/j. chb.2020.106279
Watzka, B., Hoyer, C., Ertl, B. \& Girwidz, R. (2021). Wirkung visueller und auditiver Hinweise auf die visuelle Aufmerksamkeit und Lernergebnisse beim Einsatz physikalischer Lernvideos. Unterrichtswissenschaft. https://doi.org/10.1007/s42010-021-001 18-7

Weinberger, A. Ertl, B., Fischer, F. \& Mandl, H. (2005). Epistemic and social scripts in computer-supported collaborative learning. Instructional Science, 33, 1 - 30. https://doi.org/10.1007/s11251004-2322-4

Xie, H., Mayer, R., Wang, F. \& Zhou, Z. (2019). Coordinating visual and auditory cueing in multimedia learning. Journal of Educational Psychology, 111(2), 235 -255. https://doi.org/10.1037/ edu0000285

Xie, H., Wang, F., Hao, Y., Chen, J., An, J., Wang, Y. \& Liu, H. (2017). The more total cognitive load is reduced by cues, the better retention and transfer of multimedia learning: A meta-analysis and two meta-regression analyses. PLOS ONE, 12(8). https://doi. org/10.1371/journal.pone.0183884

Yang, F.-Y., Chang, C.-Y., Chien, W.-R., Chien, Y.-T. \& Tseng, Y.-H. (2013). Tracking learners' visual attention during a multimedia presentation in a real classroom. Computers \& Education, 62, 208 -220. https://doi.org/10.1016/j.compedu.2012.10.009

\section{Historie}

Manuskript eingereicht: 20.07.2020

Manuskript nach Revision angenommen: 03.09.2021

Onlineveröffentlichung: 30.09.2021

\section{Danksagung}

Wir danken Dr. Andras Csanadi, Marcus Seichter, M. A., Patrick Pecher, M.A., Sophie Hendreich, B.A. und Erik Schmidtchen für ihre Unterstützung in der Studie und ihre konstruktiven Beiträge zur Diskussion dieses Projekts.

\section{Förderung}

Open-Access-Veröffentlichung ermöglicht durch die Universität der Bundeswehr München.

\section{ORCID}

Maleen Hurzlmeier

(iD) https://orcid.org/0000-0001-6006-4071

\author{
Maleen Hurzlmeier \\ Universität der Bundeswehr München \\ Fakultät für Humanwissenschaften \\ Professur für Lernen und Lehren mit Medien \\ Werner-Heisenberg-Weg 39 \\ 85577 Neubiberg \\ Deutschland \\ maleen.hurzlmeier@unibw.de
}

